\title{
The width of the $\omega$ meson in the nuclear medium
}

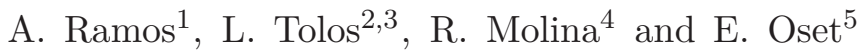 \\ 1 Departament d'Estructura i Constituents de la Matèria and Institut de Ciències del Cosmos, \\ Universitat de Barcelona, Martí i Franquès 1, E-08028 Barcelona, Spain \\ 2 Instituto de Ciencias del Espacio (IEEC/CSIC) Campus Universitat Autònoma de Barcelona, \\ Facultat de Ciències, Torre C5, E-08193 Bellaterra (Barcelona), Spain \\ ${ }^{3}$ Frankfurt Institute for Advanced Studies (FIAS). Johann Wolfgang Goethe \\ University. Ruth-Moufang-Str. 1. 60438 Frankfurt am Main. Germany \\ ${ }^{4}$ Research Center for Nuclear Physics (RCNP), \\ Mihogaoka 10-1, Ibaraki 567-0047, Japan \\ ${ }^{5}$ Departamento de Física Teórica and IFIC, \\ Centro Mixto Universidad de Valencia-CSIC, \\ Institutos de Investigación de Paterna, \\ Aptdo. 22085, E-46071 Valencia, Spain
}

(Dated: November 7, 2013)

\begin{abstract}
We evaluate the width of the $\omega$ meson in nuclear matter. We consider the free decay mode of the $\omega$ into three pions, which is dominated by $\rho \pi$ decay, and replace the $\rho$ and $\pi$ propagators by their medium modified ones. We also take into account the quasielastic and inelastic processes induced by a vector-baryon interaction dominated by vector meson exchange, as well as the contributions coming from the $\omega \rightarrow K \bar{K}$ mechanism with medium modified $K, \bar{K}$ propagators. We obtain a substantial increase of the $\omega$ width in the medium, reaching a value of $129 \pm 10 \mathrm{MeV}$ at normal nuclear matter density for an $\omega$ at rest, which comes mainly from $\omega N \rightarrow \pi \pi N, \omega N N \rightarrow \pi N N$ processes associated to the dominant $\omega \rightarrow \rho \pi$ decay mode. The value of the width increases moderately with momentum, reaching values of around $200 \mathrm{MeV}$ at $600 \mathrm{MeV} / \mathrm{c}$.

PACS numbers: 11.80.Gw,13.75.-n,14.40.Cs,21.65.+f
\end{abstract}




\section{INTRODUCTION}

The interaction of vector mesons with nuclei is a subject that has attracted much attention. The large amount of work, experimental and theoretical, has been reviewed extensively in [1] and more recently in [2, 3]. Another recent review [4], reporting on progress done using the local hidden gauge theory for the interaction of vectors [5 8], has brought a new perspective into this topic.

Although originally there were some hopes that the mass of the vector mesons would be drastically reduced in the nuclear medium, devoted studies concluded that this was not the case [9 15] and recent experiments have confirmed the results of these calculations [2, 3, 16, 17].

In addition to the emblematic $\rho$ meson, one of the vector mesons which has been more thoroughly investigated experimentally is the $\omega$ meson. Apart from investigations using heavy ions, other reactions, easier to interpret, have been carried out using proton beams on nuclei at KEK [18 20] or photonuclear reactions looking for dileptons in the final state [17, 21]. However, one of the most detailed experiments on the $\omega$ properties in the nucleus has been done at ELSA (Bonn), using photoproduction on nuclei and detecting the $\omega$ through its $\pi^{0} \gamma$ decay mode [22]. Originally, the analysis of the experimental results led the authors of 22] to claim the first observation of the in-medium modifications of the $\omega$ meson mass, with a reduction of about $100 \mathrm{MeV}$ at normal nuclear matter density. Yet, it was shown in [23] that the results of [22] were tied to a particular choice of background. A thorough study of the background processes in a revised analysis of the experiment 24] concluded that the experiment was not sensitive enough to possible changes of the in-medium $\omega$ mass. Preliminary results from the momentum distribution appear to exclude shifts of the mass by $16 \%[25]$.

Simultaneously, the possibility that some signal reported in [26, 27] could indicate the formation of a $\omega$ meson bound state in nuclei was ruled out, showing that the double hump structure observed in the experiment was due to uncorrelated $\pi^{0} \gamma$ production events together with the $\omega$ production and subsequent $\pi^{0} \gamma$ decay, which scaled differently with growing nuclear mass [28]. The expectations that the use of the mixed events method to separate the background from the signal could solve the problem [29] were also shown unproductive in [30], due to the fast exponential fall of the mass distribution which made inapplicable this otherwise successful tool.

In summary, the thorough experimental and theoretical work along these reactions led to clarify the issue and set warnings for the analysis of similar reactions used to extract medium modifications of vector meson properties.

Yet, there is some physical information that survived the close scrutiny of the former works, and this is the large width of the $\omega$ in the medium found in [31] and also studied in [23]. The CBELSA/TAPS collaboration reported a width of $130-150 \mathrm{MeV}$ at normal nuclear matter density 31], while the analysis based on a Monte Carlo simulation taking into account the possible reactions in the experimental set-up in the vicinity of the $\omega$ meson produced a distribution compatible with a width of the order of $100 \mathrm{MeV}[23]$. The theoretical understanding of this large width is a challenge that we take in the present work.

The theoretical determination of the $\omega$ properties in the medium has been addressed in numerous studies [32 53] where the attention was centered, mostly, in the change of mass. The obtained mass shifts split nearly equally into attractive and repulsive ones, and range from an attraction of the order of 100-200 MeV [35, 37], through no changes in the mass [47, 48], to a net repulsion of the order of $50 \mathrm{MeV}$ [43]. As for the in-medium width of a $\omega$ meson at rest, in [33], where the three pion decay mode is explicitly considered, it is found to increase by an order of magnitude at normal nuclear saturation density as compared to the free width, while in a subsequent revision of the model [37] a value of about $40 \mathrm{MeV}$ is reported. A similar result was also reported in [38], while the width was found to be around $60 \mathrm{MeV}$ in [46] and [49]. All these studies point to a considerable increase of the $\omega$ width in the medium with respect to its free width. 
Recently, the use of the hidden gauge theory to deal with the interaction of vector mesons with baryons, using a unitary approach with coupled channels, has brought new light into the subject since it interprets some baryonic resonances as being dynamically generated by this interaction [54, 55]. This opens a new framework to study the interaction of vector mesons with nuclei, as done recently for the $\bar{K}^{*}$ [56], where a spectacular increase of the width of the $\bar{K}^{*}$ in nuclei, of around $250 \mathrm{MeV}$ at nuclear saturation density, was found. With this perspective, we evaluate in this work the $\omega$ width in nuclear matter, exploiting the analogies with the $\bar{K}^{*}$ meson and addressing other decay channels specific to the $\omega$ meson tied to its decay into three pions.

The paper is organized as follows. The formalism employed is described in Sect. II, where the Lagrangians needed for the study of the $\omega$ properties in the medium are presented. In the next subsections, the expressions for the $\omega$ self-energy in nuclear matter corresponding to the different decay channels explored in this work are derived. Our results are discussed in Section III and some concluding remarks are given in Sect. IV.

\section{FORMALISM FOR VECTOR MESON INTERACTIONS}

A free $\omega$ meson decays predominantly into three pions, most of the strength being associated to the process $\omega \rightarrow \rho \pi$ with the subsequent decay of the $\rho$ meson into two pions. Since $m_{\omega}<m_{\rho}+m_{\pi}$, the mechanism proceeds through the tail of the $\rho$-meson distribution, as a result of which the $\omega$ width is relatively small, $\Gamma_{\omega}^{(0)}=8.49 \pm 0.08 \mathrm{MeV}$, with $89.2 \%$ of this value corresponding to the $3 \pi$ decay channel [57]. The situation may change drastically in the nuclear medium, since any of the decay pions can be absorbed by a nucleon, exciting a so-called particle-hole excitation and opening the allowed phase-space considerably. In addition, other mechanisms not forbidden by QCD symmetries but energetically closed in free space, such as $\omega \rightarrow K \bar{K}$, may also contribute when the medium modifications of the $\bar{K}$ and $K$ mesons are incorporated. One should also consider the modification of the $\omega$ properties associated to its quasielastic collisions with the nucleons in the medium or other inelastic processes not incorporated by the previously mentioned absorption mechanisms.

In this section we describe how the different contributions to the $\omega$ self-energy explored in this work are obtained. We start presenting the Lagrangians describing the interaction of the $\omega$ meson with other mesons (vectors and pseudoscalars) and with baryons of the ground-state octet. The transition $\omega \rightarrow \rho \pi$, depicted generically in Fig. 1(a), is obtained from the vector meson dominance (VMD) Lagrangians involving the anomalous $V V P$ term described in [58]

$$
\mathcal{L}_{V V P}=\frac{G}{\sqrt{2}} \epsilon^{\mu \nu \alpha \beta}\left\langle\partial_{\mu} V_{\nu} \partial_{\alpha} V_{\beta} P\right\rangle
$$

where $P$ is the matrix of pseudoscalar fields

$$
P=\left(\begin{array}{ccc}
\frac{1}{\sqrt{2}} \pi^{0}+\frac{1}{\sqrt{6}} \eta_{8} & \pi^{+} & K^{+} \\
\pi^{-} & -\frac{1}{\sqrt{2}} \pi^{0}+\frac{1}{\sqrt{6}} \eta_{8} & K^{0} \\
K^{-} & \bar{K}^{0} & -\frac{2}{\sqrt{6}} \eta_{8}
\end{array}\right),
$$

$V_{\mu}$ is the matrix of vector mesons of the nonet of the $\rho$

$$
V_{\mu}=\left(\begin{array}{ccc}
\frac{\rho^{0}}{\sqrt{2}}+\frac{\omega}{\sqrt{2}} & \rho^{+} & K^{*+} \\
\rho^{-} & -\frac{\rho^{0}}{\sqrt{2}}+\frac{\omega}{\sqrt{2}} & K^{* 0} \\
K^{*-} & \bar{K}^{* 0} & \phi
\end{array}\right)_{\mu}
$$




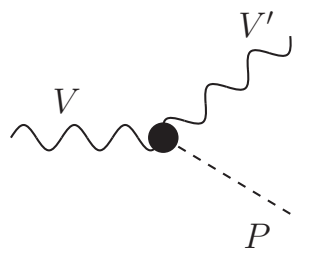

(a)

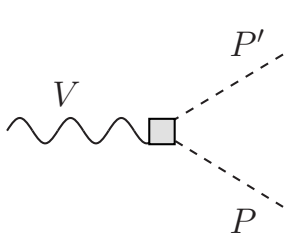

(b)

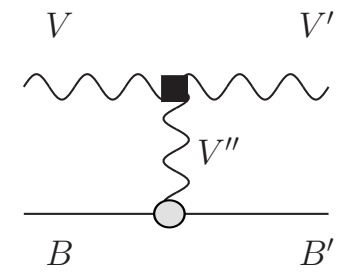

(c)

FIG. 1: Diagrams depicting the vertices needed in the evaluation of the $\omega$ meson self-energy: the anomalous $V V P$ term (a), the $V P P$ term (b), and the $V B \rightarrow V^{\prime} B^{\prime}$ amplitude mediated by t-channel vector-meson exchange.

and the \langle\rangle symbol represents the trace in $\mathrm{SU}(3)$ space. The coupling constant of the $V V P$ Lagrangian is $G=\frac{3 g^{\prime 2}}{4 \pi^{2} f}$, where $g^{\prime}=-\frac{G_{V} M_{V}}{\sqrt{2} f^{2}}$ [58], with $f=f_{\pi}=93 \mathrm{MeV}$ and $M_{V}$ an appropriate vector meson mass, which can be taken equal to the mass of the $\rho$ meson. The value of $G_{V}$ can be adjusted to $\rho \rightarrow \pi \pi$ decay $\left(G_{V}=69 \mathrm{MeV}\right)$, to the pion charged radius $\left(G_{V}=55 \mathrm{MeV}\right)$ or can be fixed invoking consistency with QCD asymptotic behavior $\left(G_{V}=f / \sqrt{2}\right)[59$, 60]. In the latter case one obtains $G=\frac{3 m_{\rho}^{2}}{16 \pi^{2} f^{3}}=14 \mathrm{GeV}^{-1}$. Alternatively, one can also use the local hidden gauge approach, which in its version of full vector meson dominance also leads to the same result [8, 61].

The formalism of the hidden gauge interaction for vector mesons, which we take from [5, 6] (see also [62] for a practical set of Feynman rules), provides the interaction of vector mesons amongst themselves

$$
\mathcal{L}_{I I I}=-\frac{1}{4}\left\langle V_{\mu \nu} V^{\mu \nu}\right\rangle
$$

where $V_{\mu \nu}$ is given by

$$
V_{\mu \nu}=\partial_{\mu} V_{\nu}-\partial_{\nu} V_{\mu}-i g\left[V_{\mu}, V_{\nu}\right]
$$

with the coupling of the theory given by $g=M_{V} / 2 f$. This Lagrangian gives rise to a three vector vertex

$$
\mathcal{L}_{I I I}^{(3 V)}=i g\left\langle\left(\partial_{\mu} V_{\nu}-\partial_{\nu} V_{\mu}\right) V^{\mu} V^{\nu}\right\rangle=i g\left\langle\left(V^{\mu} \partial_{\nu} V_{\mu}-\partial_{\nu} V_{\mu} V^{\mu}\right) V^{\nu}\right\rangle
$$

needed for the evaluation of the $V B \rightarrow V^{\prime} B^{\prime}$ interacting terms mediated by t-channel vector meson exchange, as seen in Fig. 1(c), which will describe, after unitarization, the elastic $\omega N \rightarrow \omega N$ interaction and the inelastic transitions to the related coupled channels. The Lagrangian describing the baryon-baryon-vector vertex involved in the $V B \rightarrow V^{\prime} B^{\prime}$ amplitudes is given by [33, 63]

$$
\mathcal{L}_{B B V}=g\left(\left\langle\bar{B} \gamma_{\mu}\left[V^{\mu}, B\right]\right\rangle+\left\langle\bar{B} \gamma_{\mu} B\right\rangle\left\langle V^{\mu}\right\rangle\right),
$$


(a)

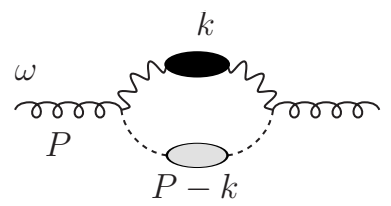

(b)

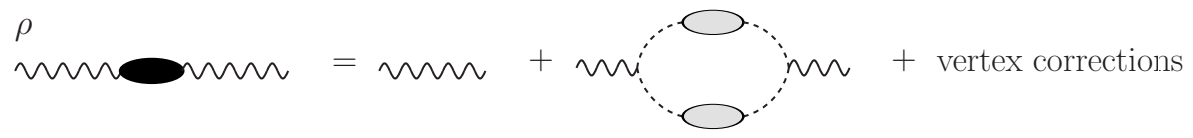

(c)
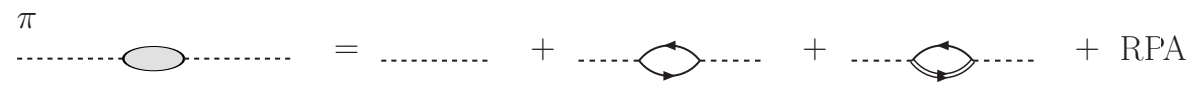

FIG. 2: Diagrammatic representation of the $\omega$ meson self-energy coming from its decay into the $\rho \pi$ mode (a), where the $\rho$ meson decays into two pions (b) and the $\pi$ propagator is dressed by its coupling to particle-hole and $\Delta$-hole excitations including short-range correlation effects (c).

where $B$ stands for the matrix of the baryon octet [64, 65].

Finally, the coupling of the vector to pseudoscalar mesons depicted in Fig. 1(b), needed for evaluating the $\omega \rightarrow \bar{K} K$ transition, is also provided by the hidden gauge Lagrangian as

$$
\mathcal{L}_{V P P}=-i g\left\langle\left[P, \partial_{\mu} P\right] V^{\mu}\right\rangle .
$$

\section{A. $\omega$ self-energy from $\rho \pi$ and uncorrelated $\pi \pi \pi$}

Let us consider first the contributions to the $\omega$ width coming from its decay into the $\rho \pi$ mode in the nuclear medium. The self-energy for this process is depicted diagrammatically in Fig. 2(a), where the $\rho$ - and $\pi$-meson lines correspond to their medium propagators shown in Figs. 2(b) and (c), respectively.

From the $V V P$ Lagrangian, 1, we obtain the following $\omega \rho \pi$ interaction vertex:

$$
-i T=i \mathcal{L}_{\omega \rho \pi}=i G \epsilon^{\mu \nu \alpha \beta} \partial_{\mu} \omega_{\nu} \partial_{\alpha} \rho_{\beta} \pi=i G \epsilon^{\mu \nu \alpha \beta} P_{\mu} \epsilon_{\nu} k_{\alpha} \epsilon_{\beta}^{\prime},
$$

where $P$ and $k$ are the four-momenta of the $\omega$ and $\rho$ mesons, respectively. The contribution to the $\omega$ self-energy coming from the diagram of Fig. 22(a) can then be written as:

$$
\begin{aligned}
-i \Pi_{\omega \rightarrow \rho \pi}(\rho, P)= & \int \frac{d^{4} k}{(2 \pi)^{4}} i G \epsilon^{\mu \nu \alpha \beta} P_{\mu} \epsilon_{\nu} k_{\alpha} \epsilon_{\beta}^{\prime} i G \epsilon^{\mu^{\prime} \nu^{\prime} \alpha^{\prime} \beta^{\prime}} P_{\mu^{\prime}} \epsilon_{\nu^{\prime}} k_{\alpha^{\prime}} \epsilon_{\beta^{\prime}}^{\prime} \\
& \times i D_{\rho}\left(\rho, k^{0}, \vec{k}\right) i D_{\pi}\left(\rho, P^{0}-k^{0}, \vec{P}-\vec{k}\right)
\end{aligned}
$$

where $D_{\rho}$ and $D_{\pi}$ stand, respectively, for the $\rho$ and $\pi$ propagators. Working out the index contractions and using the spectral decomposition of the meson propagators,

$$
D_{x}\left(\rho, q^{0}, \vec{q}\right)=\int_{0}^{\infty} \frac{d \omega}{\pi}(-2 \omega) \frac{\operatorname{Im} D_{x}(\rho, \omega, \vec{q})}{\left(q^{0}\right)^{2}-\omega^{2}+i \varepsilon} \quad \text { with } x=\rho, \pi,
$$

Eq. (10) reduces to

$$
\begin{aligned}
\Pi_{\omega \rightarrow \rho \pi}(\rho, P)= & \int \frac{d^{3} k}{(2 \pi)^{3}} \int_{0}^{\infty} \frac{d \omega}{\pi} \int_{0}^{\infty} \frac{d \omega^{\prime}}{\pi}\left[\frac{2 G^{2}\left\{(k P)^{2}-P^{2} k^{2}\right\}}{P^{0}-\omega-\omega^{\prime}+i \varepsilon}-\frac{2 G^{2}\left\{\left(k^{\prime} P\right)^{2}-P^{2} k^{2}\right\}}{P^{0}+\omega+\omega^{\prime}-i \varepsilon}\right] \\
& \times \operatorname{Im} D_{\rho}(\rho, \omega, \vec{k}) \operatorname{Im} D_{\pi}\left(\rho, \omega^{\prime}, \vec{P}-\vec{k}\right)
\end{aligned}
$$


with $P=\left(P^{0}, \vec{P}\right), k=(\omega, \vec{k}), k^{\prime}=(-\omega, \vec{k})$, after integrating appropriately over $k^{0}$ and omitting the polarization dependence $\vec{\epsilon} \vec{\epsilon}^{\prime}$. For practical purposes, one can take $k^{\prime} \simeq k$ in the last term of Eq. (12), which contributes very little due to the much larger denominator, and then the expression can be written more compactly as

$$
\begin{aligned}
\Pi_{\omega \rightarrow \rho \pi}(\rho, P)= & \int \frac{d^{3} k}{(2 \pi)^{3}} \int_{0}^{\infty} \frac{d \omega}{\pi} \int_{0}^{\infty} \frac{d \omega^{\prime}}{\pi} 2 G^{2}\left\{(k P)^{2}-P^{2} k^{2}\right\} \frac{2\left(\omega+\omega^{\prime}\right)}{\left(P^{0}\right)^{2}-\left(\omega+\omega^{\prime}\right)^{2}+i \varepsilon} \\
& \times \operatorname{Im} D_{\rho}(\rho, \omega, \vec{k}) \operatorname{Im} D_{\pi}\left(\rho, \omega^{\prime}, \vec{P}-\vec{k}\right) .
\end{aligned}
$$

Note that Eqs. (12) and (13) contain a factor of 3 accounting for the three different charged channels, $\rho^{+} \pi^{-}, \rho^{0} \pi^{0}$ and $\rho^{-} \pi^{+}$, an aspect that will be discussed later. The in-medium $\pi$ and $\rho$ propagators are obtained from their corresponding self-energy through the Dyson equation:

$$
D_{x}\left(\rho, q^{0}, \vec{q}\right)=\frac{1}{\left(q^{0}\right)^{2}-\vec{q}^{2}-m_{x}^{2}-\Pi_{x}\left(\rho, q^{0}, \vec{q}\right)} \quad \text { with } x=\rho, \pi .
$$

The pion self-energy, $\Pi_{\pi}\left(\rho, q^{0}, \vec{q}\right)$, is strongly dominated by the $p$-wave coupling to particle-hole $(p h)$ and $\Delta$-hole $(\Delta h)$ components, displayed in Fig. 2(c), and also contains a small repulsive $s$-wave contribution that takes over at small momenta, as well as contributions from $2 p$ - $2 h$ excitations, which account for two-nucleon pion absorption processes. The pion self-energy also accounts for the effect of repulsive, spin-isospin $N N$ and $N \Delta$ short range correlations [66], which are incorporated through a RPA summed Landau-Migdal type interaction, and implements the usual monopole form factor at each $\pi N N$ and $\pi N \Delta$ vertex, namely $F_{\pi}\left(\vec{q}^{2}\right)=\left(\Lambda_{\pi}^{2}-m_{\pi}^{2}\right) /\left[\Lambda_{\pi}^{2}-q^{02}+\vec{q}^{2}\right]$, with $\Lambda_{\pi}=1200 \mathrm{MeV}$, as needed in the empirical study of $N N$ interactions [67]. The details of the pion self-energy employed in this work can be found in Refs. [68, 69].

This pion self-energy is employed in Ref. [13] to obtain, via the diagrams of Fig. 2(b), the inmedium $\rho$ self-energy and the corresponding in-medium propagator. The model also implements vertex corrections and the effect of resonance-hole excitations, finding that, at normal nuclear matter density $\rho_{0}=0.17 \mathrm{fm}^{-3}$, the $\rho$ meson mass does not change and its width is increased by one third with respect to its free value, $\Gamma_{\rho}^{\text {free }}=149.4 \mathrm{MeV}$. To simplify our calculations, we parametrize the results of Ref. [13] in terms of a simple width function that depends on the nuclear density and is corrected by the kinematical factors associated to the fact that we are dealing with an off-shell $\rho$ meson with invariant mass squared $s=\omega^{2}-\vec{k}^{2}$. Thus,

$$
\Gamma_{\rho}(\rho, s)=\Gamma_{\rho}^{(0)}(s)+\Delta \Gamma_{\rho}(\rho, s)
$$

where

$$
\Gamma_{\rho}^{(0)}(s)=\Gamma_{\rho}^{\mathrm{free}} \frac{m_{\rho}^{2}}{s} \frac{\bar{k}^{3}(s)}{\bar{k}_{\mathrm{on}}^{3}}
$$

and

$$
\Delta \Gamma_{\rho}(\rho, s)=0.33 \Gamma_{\rho}^{\text {free }} \frac{\rho}{\rho_{0}} \frac{m_{\rho}^{2}}{s} \frac{k^{\prime 3}(s)}{k_{\text {on }}^{\prime 3}} .
$$

The momenta appearing in the former equations are given by

$$
\begin{gathered}
\bar{k}(s)=\frac{\lambda^{1 / 2}\left(s, m_{\pi}^{2}, m_{\pi}^{2}\right)}{2 \sqrt{s}} \theta\left(\sqrt{s}-2 m_{\pi}\right), \quad \bar{k}_{\text {on }}=\bar{k}\left(m_{\rho}^{2}\right), \\
k^{\prime}(s)=\frac{\lambda^{1 / 2}\left(s, m_{\pi}^{2}, 0\right)}{2 \sqrt{s}} \theta\left(\sqrt{s}-m_{\pi}\right), \quad k_{\text {on }}^{\prime}=k^{\prime}\left(m_{\rho}^{2}\right),
\end{gathered}
$$


where $\lambda(x, y, z)=x^{2}+y^{2}+z^{2}-2 x y-2 y z-2 z x$ is the Källen function. Note that the expression for $k^{\prime}$ reflects the fact that the in-medium $\rho$ meson decays, in first approximation, into a $\pi$ meson and a $p h$ excitation of essentially zero energy. The $\rho$ meson self-energy needed for the evaluation of the in-medium $\rho$ propagator in Eq. (13) is then given by

$$
\Pi_{\rho}(\rho, k)=-i m_{\rho} \Gamma_{\rho}\left(\rho, \omega^{2}-\vec{k}^{2}\right) .
$$

This approach of parametrizing the $\rho$ spectral function in terms of simply increasing the $\rho$ width has been employed in some experimental analyses. However, it misses the bump in the $\rho$ spectral function at low invariant masses due to the $N^{*}(1520) h$ excitation [10, 13], a region of the $\rho$ spectral function that plays a relevant role in the $\omega$ self-energy as shown recently in [70]. On the other hand, recent developments on the vector-baryon interaction using unitarity in coupled channels and input from the local hidden gauge approach [55, 71, 72] are able to provide the $\rho N$ scattering amplitude, while generating dynamically some resonances at the same time, such as the $N^{*}(1520)$ and the $N^{*}(1700)$ with $J^{P}=3 / 2^{-}$[72] and one related to the $N^{*}(1650)$ with $J^{P}=1 / 2^{-}[55$, , 71]. The isospin $I=3 / 2$ amplitudes are also evaluated in [55], although they do not present resonant structures (some resonances are generated from the interaction of vector mesons with the decuplet of baryons [54] but they do not apply here).

In view of this, we will also perform calculations dressing the $\rho$ with its low-density self-energy

$$
\Pi_{\rho}=t_{\rho N \rightarrow \rho N} \rho,
$$

where $t_{\rho N \rightarrow \rho N}$ is the $\rho N$ amplitude calculated in [55, 71, 72] averaged over total isospin $I$ and angular momentum $J$,

$$
t_{\rho N \rightarrow \rho N}=\frac{\sum_{I, J}(2 I+1)(2 J+1) t_{\rho N \rightarrow \rho N}^{I J}}{\sum_{I, J}(2 I+1)(2 J+1)},
$$

with $I=1 / 2,3 / 2$ and $J=1 / 2,3 / 2$.

In Fig. 3 we show results for the $\rho$ spectral function obtained from three prescriptions for the $\rho$ self-energy:

(a) dash-dotted line: the phenomenological width given by Eqs. (15)-(17),

(b) dashed line: employing the amplitude $t_{\rho N \rightarrow \rho N}$ obtained in Refs. [55, 71, 72] from a coupled channel unitary approach with input from the local hidden gauge lagrangians, but replacing the $I=1 / 2, J^{P}=3 / 2^{-}$contribution by the $N^{*}(1520) N^{-1}$ term of Ref. [13] that is parametrized as

$$
t^{1 / 2,3 / 2^{-}}=\frac{\tilde{g}_{N^{*} N \rho}^{2}}{\sqrt{s^{\prime}}-M_{R}+\mathrm{i} \Gamma / 2},
$$

where $M_{R}=1520 \mathrm{MeV}, \Gamma=150 \mathrm{MeV}$ and $\tilde{g}_{N^{*} N \rho}=7.73$, a value rather similar to the one obtained in [72], $\left|\tilde{g}_{N^{*} N \rho}\right|=6.39$. The argument $\sqrt{s^{\prime}}$ of $t^{1 / 2,3 / 2^{-}}$is determined from the four-momentum of the $\rho, p_{\rho}=(\omega, \vec{k})$, and that of a nucleon at rest in the nucleus, $s^{\prime}=\left(p_{\rho}+p_{N}\right)^{2}=\left(\omega+m_{N}\right)^{2}-\vec{k}^{2}$, and

(c) solid line: taking the complete $t_{\rho N \rightarrow \rho N}$ from the model of Refs. [55, 71, 72]

We can see in Fig. 3 that the $\rho$ width is quite similar in all cases, but the bump from the $N^{*}(1520)$ excitation is missing in the pure phenomenological model based on Eqs. (15)-(17). This excitation has a larger strength in the $N^{*} N^{-1}$ resonance pole approach of [13] than in the microscopical model of [72], a difference that will have repercussions on the value of the $\omega$ width in the medium as will see. 


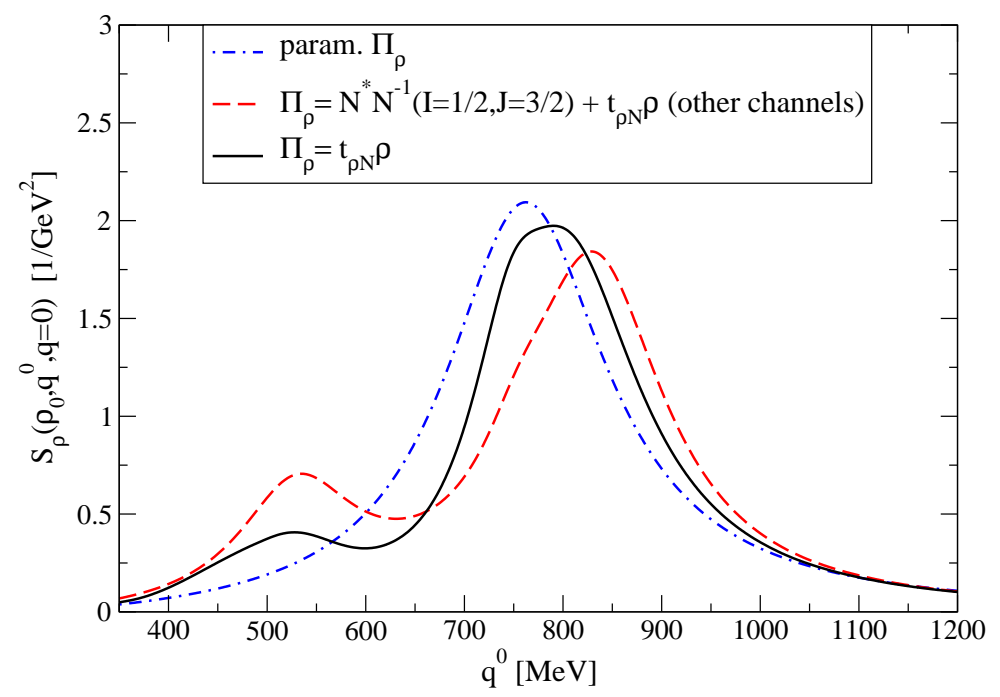

FIG. 3: The spectral function of a $\rho$-meson of zero momentum in nuclear matter at saturation density for the three prescriptions employed in this paper. Dash-dotted line: the phenomenological width given by Eqs. (15)-(17); dashed-line: employing the $t_{\rho N \rightarrow o N}$ model from the coupled channel unitary model with local hidden gauge lagrangians of Refs. [55, 71, 72] but replacing the $I=1 / 2, J^{P}=3 / 2^{-}$amplitude by the $N^{*}(1520) N^{-1}$ contribution of Ref. [13]; solid line: taking the complete $t_{\rho N \rightarrow \rho N}$ amplitude from the model of Refs. [55, 71, 72].

Finally, the in-medium $\omega$ width coming from the $\omega \rightarrow \rho \pi$ channel is obtained from

$$
\Gamma_{\omega \rightarrow \rho \pi}(\rho, P)=-\frac{\operatorname{Im} \Pi_{\omega \rightarrow \rho \pi}(\rho, P)}{P^{0}},
$$

and the corresponding in-medium width correction is

$$
\Delta \Gamma_{\omega \rightarrow \rho \pi}(\rho, P)=\Gamma_{\omega \rightarrow \rho \pi}(\rho, P)-\Gamma_{\omega \rightarrow \rho \pi}^{(0)}(P),
$$

where the free $\omega \rightarrow \rho \pi$ decay width can be obtained from the $\omega$ self-energy of Eq. (13) by replacing

$$
\begin{gathered}
\operatorname{Im} D_{\pi}\left(\rho, \omega^{\prime}, \vec{P}-\vec{k}\right) \rightarrow-\pi \delta\left(\omega^{\prime}-\omega_{\pi}(\vec{P}-\vec{k})\right) \frac{1}{2 \omega_{\pi}(\vec{P}-\vec{k})}, \\
\operatorname{Im} D_{\rho}(\rho, \omega, \vec{k}) \rightarrow \operatorname{Im} \frac{1}{\omega^{2}-\vec{k}^{2}-m_{\rho}^{2}+i m_{\rho} \Gamma_{\rho}^{(0)}\left(\omega^{2}-\vec{k}^{2}\right)} .
\end{gathered}
$$

with $\omega_{\pi}(\vec{P}-\vec{k})=\sqrt{(\vec{P}-\vec{k})^{2}+m_{\pi}^{2}}$. After performing the integration over $\vec{k}$, an appropriate change of variable allows one to write the free $\omega \rightarrow \rho \pi$ width as:

$$
\Gamma_{\omega \rightarrow \rho \pi}^{(0)}(P)=-\frac{\operatorname{Im} \Pi_{\omega \rightarrow \rho \pi}^{(0)}(P)}{P^{0}}=-\frac{G^{2}}{4 \pi^{2}} \int d \tilde{m}_{\rho}^{2} \operatorname{Im} \frac{k^{3}\left(\tilde{m}_{\rho}^{2}\right)}{\tilde{m}_{\rho}^{2}-m_{\rho}^{2}+i m_{\rho} \Gamma_{\rho}^{(0)}\left(\tilde{m}_{\rho}^{2}\right)},
$$

where $k\left(\tilde{m}_{\rho}^{2}\right)=\lambda^{1 / 2}\left(m_{\omega}^{2}, \tilde{m}_{\rho}^{2}, m_{\pi}^{2}\right) \theta\left(m_{\omega}-\tilde{m}_{\rho}-m_{\pi}\right) /\left(2 m_{\omega}\right)$.

Note that the factor of 3 in Eqs. (12)-(13) would account appropriately for the three different isospin channels, $\rho^{+} \pi^{-}, \rho^{0} \pi^{0}$ and $\rho^{-} \pi^{+}$, if the $\rho$ meson was a stable particle, since in this case the three channels would correspond to different final states that would not interfere. However, the 


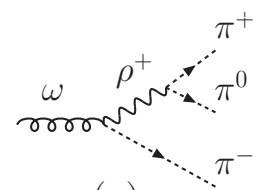

(a)

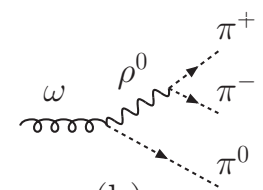

(b)

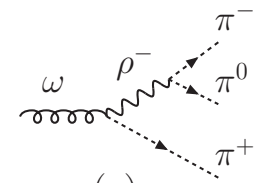

(c)

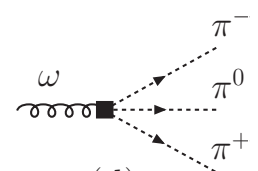

(d)

FIG. 4: Contributions to the $\omega \rightarrow \rho \pi \rightarrow \pi^{+} \pi^{0} \pi^{+}$decay process, coming from three different intermediate charged channels: $\rho^{+} \pi^{-}, \rho^{0} \pi^{0}$ and $\rho^{-} \pi^{+}$.

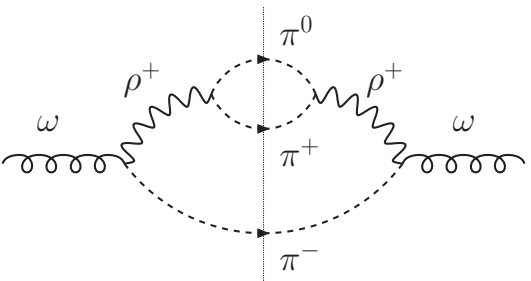

(a)

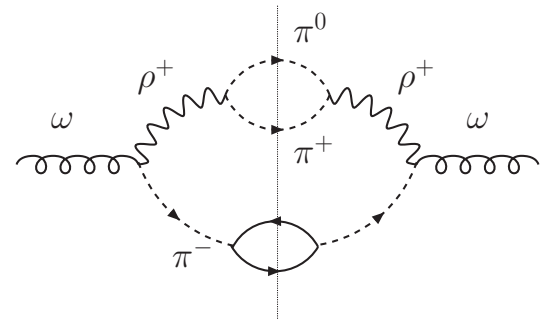

$\left(a^{\prime}\right)$

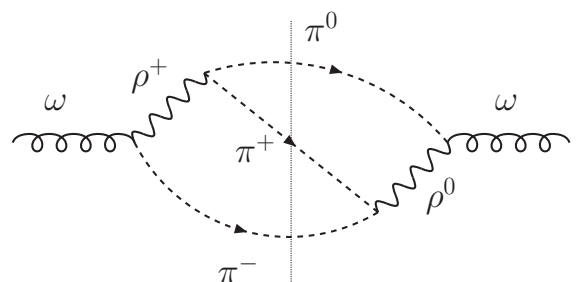

(b)

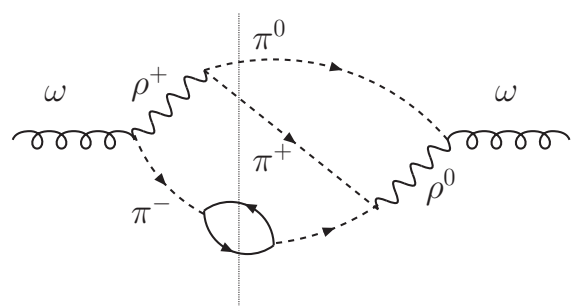

(b')

FIG. 5: Diagrammatic representation of direct and interference contributions to the $\omega$ meson self-energy coming from its decay into the $\rho \pi$ mode. Top row: free space contributions. Bottom row: in-medium contributions.

final $\rho$ meson decays into two pions, as depicted by the first three diagrams of Fig. 4. One then has identical final states coming from the three different channels that may give rise to important interference effects. This is more clearly visualized from the contributions to the $\omega$ width in free space displayed in the top row of Fig. 5, where the direct contribution of the $\rho^{+} \pi^{-}$channel is shown in diagram (a), while the interference between the $\rho^{+} \pi^{-}$and $\rho^{0} \pi^{0}$ channels is shown in diagram (b). There are a total of 9 contributions of the type shown in the top row of Fig. [5, but in our calculation we are only considering the three direct terms of type (a) and, consequently, we will miss part of the free $\omega$ width, as we will see. Since the interference terms vanish when the $\rho$ width is zero, a rough guess of their relevance can be given by $\Gamma_{\rho} / m_{\rho}$. However, when these processes occur in the medium, the situation is quite different, as can be easily understood from the diagrams displayed in the bottom row of Fig. 5. We see there that the pion emitted in the $\omega$ decay converts into a $p h$ excitation, which costs very little energy. In this case, the virtual $\rho$ meson can be left practically on-shell, enhancing the size of its propagator. For this reason, the direct diagram represented in Fig. $5\left(\mathrm{a}^{\prime}\right)$, where the two $\rho$ propagators can be practically put on-shell simultaneously, is much larger than any of the other interference diagrams, such as that in Fig. $5\left(b^{\prime}\right)$, because in this case when the $\rho$ propagator on the left carries practically the full incoming energy $m_{\omega}$, being then almost on-shell, the $\rho$ propagator on the right only carries an energy of about $m_{\omega} / 2$, being far off-shell. Therefore, the contribution of the interference terms to 
the in-medium corrections of the $\omega$ meson self-energy is essentially negligible. A more quantitative estimate can be done knowing that the average $\pi^{-}$momentum in diagrams $\left(\mathrm{a}^{\prime}\right)$ and $\left(\mathrm{b}^{\prime}\right)$ carries around $300 \mathrm{MeV} / \mathrm{c}$. One finds that the $p h$ excitation takes about $50 \mathrm{MeV}$ of energy and then the $\rho^{+}$has an invariant mass of about $665 \mathrm{MeV}$ in diagrams $\left(\mathrm{a}^{\prime}\right)$ and $\left(\mathrm{b}^{\prime}\right)$, while the $\rho^{0}$ in diagram $\left(\mathrm{b}^{\prime}\right)$ has an invariant mass around $387 \mathrm{MeV}$. The combination of the reduced $\rho^{0}$ propagator and its smaller $\pi \pi$ vertex renders the diagram $\left(\mathrm{b}^{\prime}\right)$ much less relevant compared to $\left(\mathrm{a}^{\prime}\right)$ than diagram (b) is with respect to (a), since in this latter case the invariant masses of the propagators are much closer, as one finds, analogously, taking an estimated average $\pi^{-}$momentum of $200 \mathrm{MeV} / \mathrm{c}$.

In order to obtain the $\omega$ width, we need to take a value for the coupling constant $G$. Following the arguments given in the recent work of Ref. [73], this coupling can be determined from different sources. Their fit to vector meson radiative decays considering that the photon emission is mediated by neutral vector mesons favors a value $G=11.9 \pm 0.2 \mathrm{GeV}^{-1}$, which is supported by the agreement between $V M D$ and low energy theorems for $\pi^{0} \rightarrow \gamma \gamma$ decay. Using this value for the coupling constant $G$, a free $\omega$ width of $\Gamma_{\omega \rightarrow \rho \pi}^{(0)}=4.4 \mathrm{MeV}$ is reported in [73]. In the present work we obtain, from equation Eq. (26), a value of $2.8 \mathrm{MeV}$, the difference coming precisely from the interference terms not considered here. The lack of agreement with the experimental $\omega$ width from its threepion decay channel, $\Gamma_{\omega \rightarrow 3 \pi}^{\exp }=7.57 \pm 0.07 \mathrm{MeV}$ [57], drove the authors of [73] to complement their model with a contact term, as that of the diagram of Fig. 4(d), or with contributions from higher mass meson resonances, like the $\rho^{\prime}(1450)$. The latter contributions can also be mimicked by a contact term, especially because some of the $\rho^{\prime}$ meson decay parameters are not sufficiently well known. The coupling constant of the contact term can then be adjusted so as to reproduce the free decay width of the $\omega$ into three pions. One may view the effect of the contact term as providing a background that needs to be added to the $\omega \rightarrow \rho \pi$ process. A different approach to this problem is followed in [8, 61]. In these works the local hidden gauge approach is used to determine the anomalous sector and, in addition to the $\omega \rightarrow \rho \pi \rightarrow \pi \pi \pi$ decay mode, one derives a contact term given in terms of the parameters $c_{1}, c_{2}$ and $c_{3}$, which one can determine under the assumption of vector meson dominance. Here, we have chosen to follow the more phenomenological approach of [73].

An straightforward implementation of the contact term is obtained by the following replacement

$$
G^{2} \operatorname{Im} D_{\rho}(\rho, \omega, \vec{k}) \rightarrow A \operatorname{Im}\left\{\frac{1}{\omega^{2}-\vec{k}^{2}-m_{\rho}^{2}-m_{X}^{2}-i m_{\rho} \Gamma_{\rho}\left(\rho, s=\omega^{2}-\vec{k}^{2}\right)}\right\}
$$

in Eq. (13), taking $m_{X} \rightarrow \infty$. This substitution implements a propagator having a practically constant real part and an imaginary part that incorporates the correct two-pion phase-space in the free case or the medium corrected phase-space at finite density.

The dynamical arguments for the negligible effect of in-medium interferences in the case of the $\omega \rightarrow \rho \pi$ process, based on the $\rho$ propagator being practically on-shell, are no longer valid for the terms involving the contact term (direct contact term contributions and their interference with the $\rho \pi$ terms). We then assume these contact-term interferences to have a similar size in free space and in the medium. Therefore, interpreting the constant $A$ as containing effectively the effect of these interferences, we adjust its value so that our calculation reproduces the strength of the free $\omega$ width not accounted for by the $\omega \rightarrow \rho \pi$ terms, namely $\Delta \Gamma_{\omega \rightarrow 3 \pi}^{\text {contact }}=\Gamma_{\omega \rightarrow 3 \pi}^{\exp }-\Gamma_{\omega \rightarrow \rho \pi}^{(0)}=7.57$ $\mathrm{MeV}-4.4 \mathrm{MeV}=3.17 \mathrm{MeV}$, where we have taken the proper value for $\Gamma_{\omega \rightarrow \rho \pi}^{(0)} \rho$ that contains the interference effects of the $\rho \pi$ mechanism [73]. An alternative option is to adjust the size of $G$ to reproduce the complete free $\omega \rightarrow \pi \pi \pi$ width directly from the $\rho \pi$ mechanism. The required value is then $G=15.7 \mathrm{GeV}^{-1}[73]$. We shall exploit both options in the results section. 

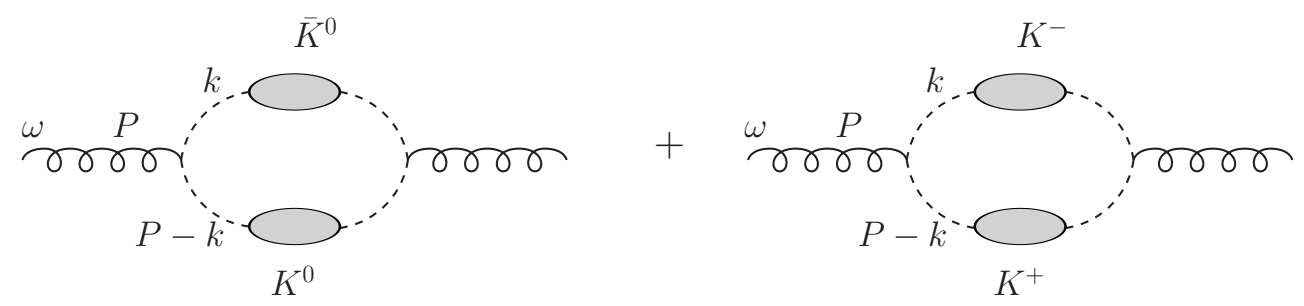

FIG. 6: The $\omega \rightarrow \bar{K} K$ channel renormalized in the nuclear medium.

\section{B. $\omega$ self-energy from $\bar{K} K$}

Analogously to the study of the $\bar{K}^{*}$ meson in the nuclear medium [56], where the decay of the $\bar{K}^{*}$ meson to $\bar{K} \pi$ is renormalized by the inclusion of the $\bar{K}$ and $\pi$ self-energies in the loop function, we consider in this section the possible modifications of the $K \bar{K}$ loop in the $\omega$ propagator in the nuclear medium as depicted in Fig. 6. The difference respect to the case of the $\bar{K}^{*}$ meson decaying into $\bar{K} \pi$ is, though, that the $\omega \rightarrow \bar{K} K$ channel is energetically closed in free space. However, due to the inclusion of the self-energy of the $\bar{K}$ meson, some processes are possible in nuclear matter.

Let us consider first the diagrams in Fig. 6] in the free case, i.e. ignoring the self-energy insertions. For the coupling of the $\omega$ meson to a $\bar{K} K$ pair, we take the Lagrangian given by Eq. (8). Both channels depicted in Fig. 6. neutral and charged, are included in the evaluation of this contribution to the $\omega$ self-energy that in the free space can be written as

$$
-i \Pi_{\omega \rightarrow K \bar{K}}^{0}(P)=4 g^{2} \int \frac{d^{4} k}{(2 \pi)^{4}} \frac{k_{\mu} \epsilon^{\mu} k_{\nu} \epsilon^{\nu}}{k^{2}-m_{\bar{K}}^{2}+i \epsilon} \frac{1}{(P-k)^{2}-m_{K}^{2}+i \epsilon} .
$$

Making the substitution:

$$
k_{\mu} k_{\nu} \epsilon^{\mu} \epsilon^{\nu} \rightarrow \frac{1}{3 P^{2}}\left\{P^{2} k^{2}-(k P)^{2}\right\} \epsilon \epsilon^{\prime} \rightarrow \frac{1}{3 P^{2}}\left\{(k P)^{2}-P^{2} k^{2}\right\} \vec{\epsilon} \vec{\epsilon}^{\prime},
$$

which for a $\omega$ meson of zero momentum equals $\vec{k}^{2} / 3$, and performing the integral in the $k^{0}$ variable, we obtain,

$$
\Pi_{\omega \rightarrow K \bar{K}}^{0}(P)=\frac{4 g^{2}}{3 P^{2}} \int \frac{d^{3} k}{(2 \pi)^{3}}\left[\frac{\omega_{K}(\vec{P}-\vec{k})+\omega_{\bar{K}}(\vec{k})}{2 \omega_{K}(\vec{P}-\vec{k}) \omega_{\bar{K}}(\vec{k})}\right] \frac{(k P)^{2}-P^{2} k^{2}}{\left(P^{0}\right)^{2}-\left[\omega_{K}(\vec{P}-\vec{k})+\omega_{\bar{K}}(\vec{k})\right]^{2}+i \varepsilon} .
$$

In the medium, we include the self-energy of the antikaon, $\Pi_{\bar{K}}\left(\rho, k^{0}, \vec{k}\right)$, and obtain the antikaon propagator

$$
D_{\bar{K}}\left(\rho, k^{0}, \vec{k}\right)=\frac{1}{\left(k^{0}\right)^{2}-\vec{k}^{2}-m_{\bar{K}}^{2}-\Pi_{\bar{K}}\left(k^{0}, \vec{k}\right)},
$$

that in the Lehmann representation reads

$$
D_{\bar{K}}\left(\rho, k^{0}, \vec{k}\right)=\int_{0}^{\infty} \frac{d \omega}{\pi}(-)\left\{\frac{\operatorname{Im} D_{\bar{K}}(\rho, \omega, \vec{k})}{k^{0}-\omega+i \eta}-\frac{\operatorname{Im} D_{K}(\rho, \omega, \vec{k})}{k^{0}+\omega-i \eta}\right\} .
$$

For the details of the antikaon self-energy and, hence, its propagator, we refer to [68, 69, 74,83$]$. Essentially, the $\bar{K}$ self-energy in symmetric nuclear matter is obtained from the antikaon-nucleon 
interaction within a chiral unitary approach. The model incorporates $s$ - and $p$-wave contributions in a self-consistent manner. The coupled-channel structure for the $s$-wave interaction includes the following channels: $\bar{K} N, \pi \Sigma, \eta \Lambda, K \Xi$ for isospin $I=0$, and $\bar{K} N, \pi \Lambda, \pi \Sigma, \eta \Sigma, K \Xi$ for isospin $I=1$. The $p$-wave contribution to the $\bar{K}$ self-energy is built up from the coupling of the $\bar{K}$ meson to $\Lambda N^{-1}, \Sigma N^{-1}$ and $\Sigma^{*} N^{-1}$ excitations.

For the kaon, due to the much weaker $K N$ interaction, we use the low-density approximation and replace $m_{K}^{2} \rightarrow m_{K}^{2}+t_{K N \rightarrow K N} \rho$, with $t_{K N \rightarrow K N} \rho=0.13 m_{K}^{2} \rho / \rho_{0}$ [84]. Thus, we can write the self-energy of the $\omega$ meson as,

$$
\begin{aligned}
-i \Pi_{\omega \rightarrow K \bar{K}}(\rho, P)= & -\frac{4 g^{2}}{3 P^{2}} \int \frac{d^{4} k}{(2 \pi)^{4}} \frac{(k P)^{2}-P^{2} k^{2}}{(P-k)^{2}-m_{K}^{2}-t_{K N \rightarrow K N} \rho} \\
& \times \int_{0}^{\infty} \frac{d \omega}{\pi}\left\{\frac{\operatorname{Im} D_{\bar{K}}(\omega, \vec{k})}{k^{0}-\omega+i \eta}-\frac{\operatorname{Im} D_{K}(\omega, \vec{k})}{k^{0}+\omega-i \eta}\right\} .
\end{aligned}
$$

As we take the physical mass of the $\omega$ meson, the real part of its in-medium self-energy must vanish at $\rho=0$. Therefore, the real part of the free $\omega$ self-energy, $\Pi_{\omega \rightarrow K \bar{K}}^{(0)}(P)$, must be subtracted to $\Pi_{\omega \rightarrow K \bar{K}}(\rho, P)$. The part of negative energy of the $\bar{K}$ propagator, represented by the last term in Eq. (32), is small. Its medium modifications are not relevant and to a very good approximation it will be cancelled by the corresponding term of the free $\omega$ self-energy. Then, after integrating over the $k^{0}$ variable, and subtracting the real part of the free $\omega \rightarrow K \bar{K}$ self-energy of Eq. (30), one gets the following correction to the $\omega$ width:

$$
\begin{aligned}
\Delta \Pi_{\omega \rightarrow K \bar{K}}(\rho, P)= & -\frac{4 g^{2}}{3 P^{2}} \int \frac{d^{3} k}{(2 \pi)^{3}}\left\{(k P)^{2}-P^{2} k^{2}\right\}\left[\int_{0}^{\infty} \frac{d \omega}{\pi} \frac{\operatorname{Im} D_{\bar{K}}(\rho, \omega, k)}{2 \tilde{\omega}_{K}(\vec{P}-\vec{k})} \frac{1}{P^{0}-\tilde{\omega}_{K}(\vec{P}-\vec{k})-\omega+i \eta}\right. \\
& \left.+\frac{1}{2 \omega_{K}(\vec{P}-\vec{k}) 2 \omega_{\bar{K}}(\vec{k})} \frac{1}{P^{0}-\omega_{K}(\vec{P}-\vec{k})-\omega_{\bar{K}}(\vec{k})+i \eta}\right] .
\end{aligned}
$$

where $\tilde{\omega}_{K}(\vec{P}-\vec{k})=\sqrt{(\vec{P}-\vec{k})^{2}+m_{K}^{2}+t_{K N \rightarrow K N} \rho}$. Because of gauge invariance of the model, it
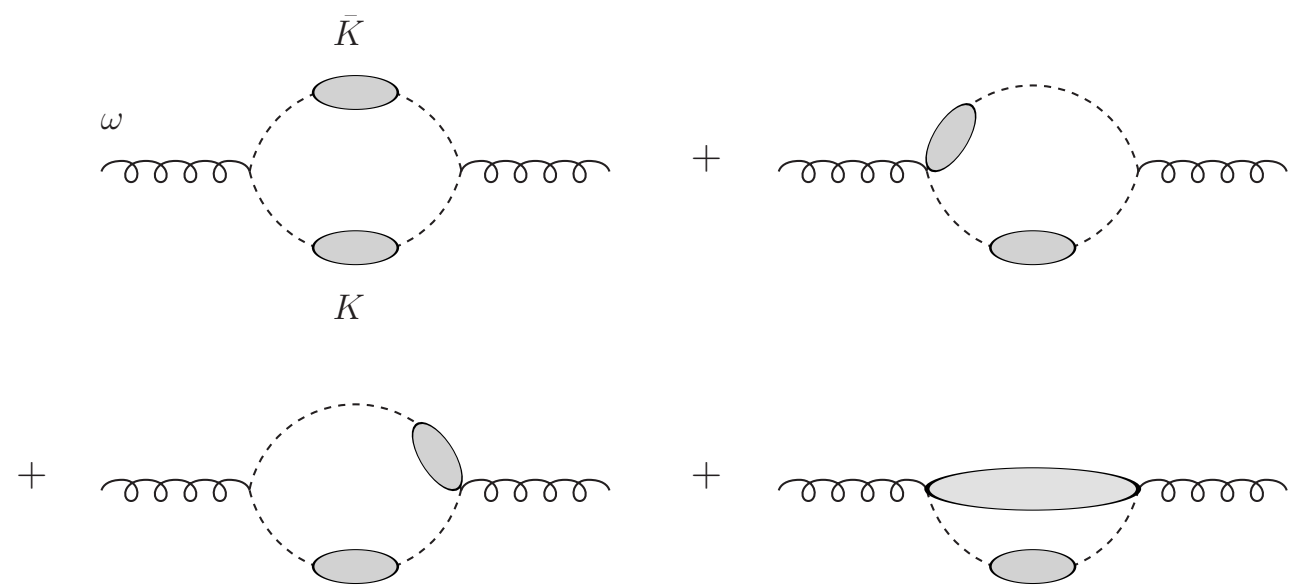

FIG. 7: Self-energy diagrams at first order in the nuclear density contributing to the decay of the $\omega$ meson in the medium.

is necessary to include vertex corrections [85, 86] associated to the last three diagrams of Fig. 0 Here we only consider vertex corrections for the antikaon. As described in Ref. [84], the vertex 
corrections are easily implemented by evaluating the diagram of Fig. 7 with the $p$-wave antikaon self-energy replaced by

$$
\Pi_{\bar{K}}^{\mathrm{p}-\text { wave }}(\rho, k) \rightarrow \frac{\Pi_{\bar{K}}^{\mathrm{p}-\text { wave }}(\rho, k)}{\vec{k}^{2}}\left(\vec{k}^{2}+\left[D_{\bar{K}}^{0}(k)\right]^{-1}+\frac{3}{4} \frac{\left[D_{\bar{K}}^{0}(k)\right]^{-2}}{\vec{k}^{2}}\right),
$$

where $D_{\bar{K}}^{0}(k)$ is the free $\bar{K}$ meson propagator.

\section{C. $\omega$ self-energy from the s-wave $\omega N$ interaction with vector mesons and baryons}

For completeness we also explore the contribution to the $\omega$ self-energy coming from the s-wave $\omega N$ interaction with vector mesons and baryons. The $\omega N$ interaction can be constructed within the hidden gauge formalism in coupled channels, as done in Ref. [55]. We proceed as in this latter reference by constructing the Feynman diagrams that lead to the vector-baryon $(V B)$ transitions $V B \rightarrow V^{\prime} B^{\prime}$ via the exchange of a vector meson, as seen in Fig. 1(c). We thus make use of the three-vector vertex Lagrangian, given by Eq. (6), as well as the Lagrangian for the coupling of vector mesons to the baryon octet given in Eq. (7). Since we are interested in studying the interaction of the $\omega$ meson in nuclear matter, we concentrate in the isospin $I=1 / 2$ sector, where we find five vector meson-baryon channels that couple to the $\omega N$ system: $\rho N, \omega N, \phi N, K^{*} \Lambda$ and $K^{*} \Sigma$.

As discussed in Ref. [55], one can proceed by neglecting the three momentum of the external vectors versus the vector mass, in a similar way as done for chiral Lagrangians under the lowmomentum approximation, and one obtains the $V B \rightarrow V^{\prime} B^{\prime}$ transition potential:

$$
V_{i j}=-C_{i j} \frac{1}{4 f^{2}}\left(k_{i}^{0}+k_{j}^{0}\right) \vec{\epsilon}_{i} \vec{\epsilon}_{j}
$$

where $k_{i}^{0}, k_{j}^{0}$ are the energies of the incoming and outgoing vector mesons, respectively, $\vec{\epsilon}_{i} \vec{\epsilon}_{j}$ is the product of their polarization vectors, and $C_{i j}$ are the symmetry coefficients [55].

The meson-baryon scattering amplitude is then obtained from the coupled-channel on-shell Bethe-Salpeter equation [74, 77]

$$
T=\left[I-V G_{V B}\right]^{-1} V,
$$

with $G_{V B}$ being the diagonal matrix of the loop functions for the meson-baryon intermediate states. We note that the iteration of diagrams implicit in the Bethe-Salpeter equation for the scattering of vector mesons with baryons implies a sum over the polarizations of the internal vector mesons which, because they are tied to the external ones through the $\vec{\epsilon}_{i} \vec{\epsilon}_{j}$ factor, leads to a correction in the $G_{V B}$ function of $\vec{q}^{2} / 3 M_{V}^{2}$ [87], which can be safely neglected in consonance with the lowmomentum approximation done in [55]. This leads to the factorization of the factor $\vec{\epsilon}_{i} \vec{\epsilon}_{j}$ for the external vector mesons also in the $T$ matrix. As a consequence, the $J^{P}=1 / 2^{-}, 3 / 2^{-}$vector-baryon scattering amplitudes are degenerate.

The loop function of a vector meson of mass $m$ and a baryon of mass $M$ with total fourmomentum $P\left(s=P^{2}\right)$ reads:

$$
G_{V B}\left(s, m^{2}, M^{2}\right)=i 2 M \int \frac{d^{4} q}{(2 \pi)^{4}} \frac{1}{(P-q)^{2}-M^{2}+\mathrm{i} \epsilon} \frac{1}{q^{2}-m^{2}+\mathrm{i} \epsilon},
$$

which is evaluated using dimensional regularization and taking a natural value of -2 for the subtraction constants at a regularization scale $\mu=630 \mathrm{MeV}$ [77]. However, the relatively large 
decay width of the $\rho$ vector meson into $\pi \pi$ pairs is incorporated in the loop function via the convolution [88]:

$$
\tilde{G}(s)=\frac{1}{N} \int_{\left(m-\Delta_{l}\right)^{2}}^{\left(m+\Delta_{r}\right)^{2}} d \tilde{m}^{2}\left(-\frac{1}{\pi}\right) \operatorname{Im} \frac{1}{\tilde{m}^{2}-m^{2}+\mathrm{i} m \Gamma^{(0)}\left(\tilde{m}^{2}\right)} G_{V B}\left(s, \tilde{m}^{2}, M^{2}\right),
$$

with

$$
N=\int_{\left(m-\Delta_{l}\right)^{2}}^{\left(m+\Delta_{r}\right)^{2}} d \tilde{m}^{2}\left(-\frac{1}{\pi}\right) \operatorname{Im} \frac{1}{\tilde{m}^{2}-m^{2}+\mathrm{i} m \Gamma^{(0)}\left(\tilde{m}^{2}\right)}
$$

being the normalization factor. The integration range around the $\rho$ mass is established by the left and right parameters $\Delta_{l}, \Delta_{r}$, taken to be a few times the free $\rho$ width, $\Gamma_{\rho}^{\text {free }}$. The energy dependent width $\Gamma^{(0)}\left(\tilde{m}^{2}\right)$ is given in Eq. (16).

In nuclear matter we should consider the contributions to the $\omega$ self-energy coming from its interactions with the nucleons in the Fermi sea. The weak interaction of $\omega$ mesons with the nucleons, as seen in Ref. [55], suggests the possibililty of extending the validity of the low-density theorem to normal nuclear matter density or beyond. Thus, we obtain the $\omega$ self-energy, depicted in Fig. 8 , by summing the $t_{\omega N \rightarrow \omega N}$ amplitude in free space over the nucleon Fermi sea, $n(\vec{k})=\theta\left(k_{F}-|\vec{k}|\right)$,

$$
\Pi_{\omega \rightarrow V B N^{-1}}(\rho, P)=\int \frac{d^{3} k}{(2 \pi)^{3}} n(\vec{k}) 4 t_{\omega N \rightarrow \omega N}\left(P^{0}+E_{N}(\vec{k}), \vec{P}+\vec{k}\right),
$$

where $P^{0}, \vec{P}$ stand for the energy and momentum of the $\omega$ in the nuclear matter rest frame, and $E_{N}(\vec{k})=\sqrt{\vec{k}^{2}+M_{N}^{2}}$ is the nucleon energy.

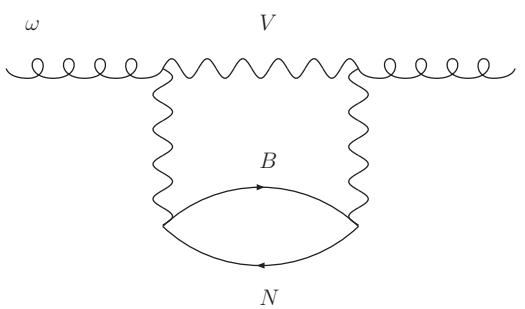

FIG. 8: Self-energy diagram coming from the s-wave $\omega N$ interaction with vector mesons and baryons

\section{General remarks}

As one can see, we have followed here a relatively unconventional approach. Some groups study the $\gamma N \rightarrow \omega N, \pi N \rightarrow \omega N, \omega N \rightarrow \pi \pi N$ reactions incorporating a set of resonances, their properties and couplings to $\gamma N$ or meson-baryon components being fitted to data, using also constraints from better known reactions, such as $\gamma N \rightarrow \pi N, \gamma N \rightarrow \pi \pi N, \pi N \rightarrow \pi \pi N$, etc. However, there are still large ambiguities, as reflected from the different results obtained by different groups or even within the same group [3, 47, 49] . Other works like that of Ref. [43] generate the resonances dynamically assuming that all baryons other than $N$ and $\Delta(1232)$ are composite meson-baryon states. The work has the advantatge of providing not only the resonances but also the couplings, thus reducing uncertainties. Yet, it also has its limitations, since not all resonances are dynamically 
generated, or at least some of them do not qualify as meson-baryon quasibound states but rather as states made out of two mesons and one baryon [89, 90]. Some also demand a subtle mixture of pseudoscalar-baryon and vector-baryon components [71, 72].

Arguments for a limited value of explicit analogies in related reactions can be found in the $\gamma N \rightarrow \pi \pi N$ process. A successful model for this reaction was developed in [94, 95] where the $\Delta$ resonance played a dominant role in a diagram as that of Fig. 9(a), which in the local hidden gauge approach is interpreted as the diagram of Fig. 9(b) plus its associated Kroll Ruderman term [72]. Note however that, while $V$ in diagram Fig. 9(b) stands in principle for the neutral vector mesons $\rho, \omega, \phi$, the $\omega$ meson does not contribute because of the G-parity forbidden $\omega \rightarrow \pi \pi$ decay, thus the link between the $\gamma N \rightarrow \pi \pi N$ and $\omega N \rightarrow \pi \pi N$ reactions is lost.

Our approach has looked in detail into the decay channels of the $\omega$ in the medium, including some which are overlooked in other works, as those studied in the two former subsections. The most important contribution, coming from $\omega \rightarrow \rho \pi$ decay, relies on an explicit calculation of the $\rho N$ scattering matrix which generates dynamically some resonances, as studied in [55, 71, 72].

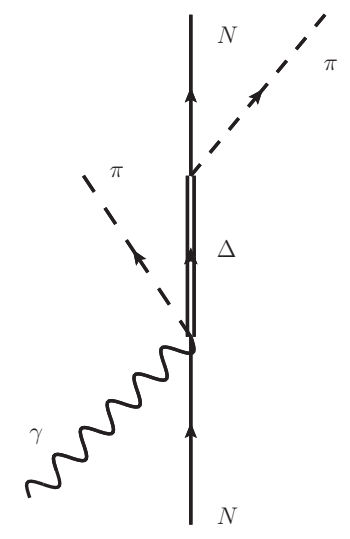

(a)

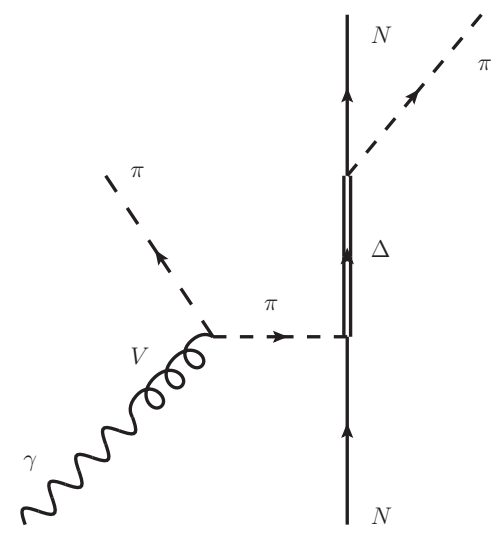

(b)

FIG. 9: Most relevant mechanism for the $\gamma N \rightarrow \pi \pi N$ reaction (a), and its interpretation within the local hidden gauge approach (b).

\section{RESULTS}

We start this section by showing our results for the contributions to the in-medium $\omega$ width coming from the $\omega \rightarrow 3 \pi$ process. We recall that, due to the coupling of the pion to $p h$ excitations in the medium, this channel would mainly correspond to absorption processes of the type $\omega N \rightarrow \pi \pi N$, together with higher order contributions in density of the type $\omega N N \rightarrow \pi N N$.

In Table \we give the in-medium width correction $\Delta \Gamma_{\omega \rightarrow \rho \pi}$ for a $\omega$ meson at rest in nuclear matter at normal saturation density employing two different models for the $\omega \rightarrow \rho \pi$ interaction and dressing the $\rho$ with the phenomenological parametrization of the width given by Eq. (17). The first model takes a $\omega \rightarrow \rho \pi$ coupling value of $G=11.9 \mathrm{GeV}^{-1}$, obtained from a fit to vector meson radiative decays [73]. Since this value of $G$ does not give the full $3 \pi$ width of a free $\omega$, the model must be complemented by a contact term, as already explained in Sect. IIA. The contribution of these two terms to the in-medium $\omega$ width correction are shown in Table \, together with their sum. The $\rho \pi$ mechanism represents about $60 \%$ of the $3 \pi$ strength. The second model corresponds to a totally different strategy, which consists of adjusting the the $\omega \rho \pi$ coupling to a value $G=15.7$ $\mathrm{GeV}^{-1}$, which reproduces the full $\omega \rightarrow 3 \pi$ free width. Interestingly, the results obtained by the two 
models are quite similar. This is a welcome feature since any other model employing a different value of $G$, when complemented by the appropriate contact-term, should produce values of the in-medium $\omega$ width correction in between the ones obtained for the two extreme models considered in this work.

\begin{tabular}{l|cc} 
& $G=11.9 \mathrm{GeV}^{-1}$ & $G=15.7 \mathrm{GeV}^{-1}$ \\
\hline$\omega \rightarrow \rho \pi$ & 54.5 & 94.9 \\
contact & 36.9 & - \\
total $\Delta \Gamma_{\omega \rightarrow 3 \pi}$ & 91.4 & 94.9 \\
\hline
\end{tabular}

TABLE I: Contributions to the in-medium correction of the $\omega$ width $\Delta \Gamma_{\omega \rightarrow 3 \pi}$ at $P^{0}=m_{\omega}$ and $\vec{P}=0$ in nuclear matter at normal saturation density, for two different $\omega \rightarrow \rho \pi$ coupling models, in the case of dressing the $\rho$ with the phenomenological parametrization of Eq. (17) and taking a pion form-factor cut-off of $\Lambda_{\pi}=1200 \mathrm{MeV}$.

Unless it is explicitly mentioned, the results shown in this section will be calculated using the effective coupling of $G=15.7 \mathrm{GeV}^{-1}$. The results of Table $\amalg$ intend to estimate how the in-medium $\omega$ width correction coming from the $\omega \rightarrow \rho \pi$ mechanism depends on the particular in-medium properties of the $\pi$ and $\rho$ mesons. We present results for three different values of the pion form-factor cut-off, $\Lambda_{\pi}=1000,1200$, and $1400 \mathrm{MeV}$, which are the appropriate ones for monopole type form factors employed in studies of the nucleon-nucleon interaction [67]. The first row gives the results obtained when only the $\pi$ meson is dressed, while the results of the next rows incorporate the additional dressing of the $\rho$ meson according to the three different approaches considered in this work: a) a simple parametrization consisting in an increase of the $\rho$ width by $33 \%$ at $\rho=\rho_{0}$ (second row), b) the approach that mimicks the results of Ref. [13] by implementing the coupling to an explicit $N^{*}(1520)$ resonance (third row) and, finally, c) a $\rho$ self-energy obtained from a $t_{\rho N \rightarrow \rho N}$ amplitude derived entirely from the hidden gauge formalism that generates the $N^{*}(1520)$ dynamically (fourth row). We observe that the in-medium pion self-energy represents

\begin{tabular}{l|ccc} 
& $\Lambda_{\pi}=1000 \mathrm{MeV}$ & $\Lambda_{\pi}=1200 \mathrm{MeV}$ & $\Lambda_{\pi}=1400 \mathrm{MeV}$ \\
\hline only $\pi$ & 69.0 & 76.5 & 79.2 \\
$\pi+\rho($ param $)$ & 85.1 & 94.9 & 98.9 \\
$\pi+\rho\left(N^{*} N^{-1}\right)$ & 128.7 & 144.5 & 153.5 \\
$\pi+\rho\left(t_{\rho N \rightarrow \rho N}\right)$ & 91.1 & 101.2 & 106.4 \\
\hline
\end{tabular}

TABLE II: Contributions to the in-medium correction of the $\omega$ width $\Delta \Gamma_{\omega \rightarrow 3 \pi}$ at $P^{0}=m_{\omega}$ and $\vec{P}=0$ in nuclear matter at normal saturation density, for three different values of the pion form-factor cut-off and three different models for the $\rho$-meson self-energy.

the most important contribution to the $\omega$ width, as already noted in [46]. Dressing the $\rho$ also adds a substantial contribution, more moderate in the case of the phenomenological model for the $\rho$ self-energy because the low-energy components of the $\rho$ spectral function play a significant role, as stressed recently in [70], where they are referred to as "space-like" contributions. This also explains that the approach using an explicit $N^{*}$ component, with more strength at low energy, gives a larger value for the in-medium $\omega$ width correction, in qualitative agreement with what is found in [70]. We have checked that, for a given model of the $\rho$ self-energy, changes in other components of the pion self-energy besides the form-factor cut-off, such as employing a constant Landau-Migdal parameter $g^{\prime}=0.6$, or implementing relativistic corrections at the $\pi N N$ and $\pi N \Delta$ 
vertices, or using a static version of the pion form factor, do not alter the results beyond the range of values shown in Table II

Adding the free decay width of the $\omega \rightarrow \rho \pi$ channel, $7.57 \mathrm{MeV}$, and dessing the $\rho$ meson with the $t_{\rho N \rightarrow \rho N}$ amplitude derived entirely from the hidden gauge lagrangians in a unitary coupled channel model, we obtain $\Gamma_{\omega}=109 \pm 10 \mathrm{MeV}$, the error being associated to variations in the parameters of the $\pi$ meson self-energy within reasonable limits.

We would like to comment on the fact that Eq. (13) also provides a real part of the $\omega$ self-energy in the medium, $\operatorname{Re} \Pi_{\omega \rightarrow \rho \pi}(\rho, P)$. When subtracting the free space contribution, $\operatorname{Re} \Pi_{\omega \rightarrow \rho \pi}(\rho=$ $0, P)$, one could obtain an estimate of the $\omega$ mass shift. However, the calculation requires to perform integrals over high momentum and energy components of the $\pi$ and $\rho$ propagators, on which one does not have a good knowledge. We have explored various strategies and have obtained $\omega$ mass shifts ranging from -100 to $100 \mathrm{MeV}$, meaning that it is not possible to obtain a sensible stable result. Therefore we focus only on discussing the medium modifications of the $\omega$ width.

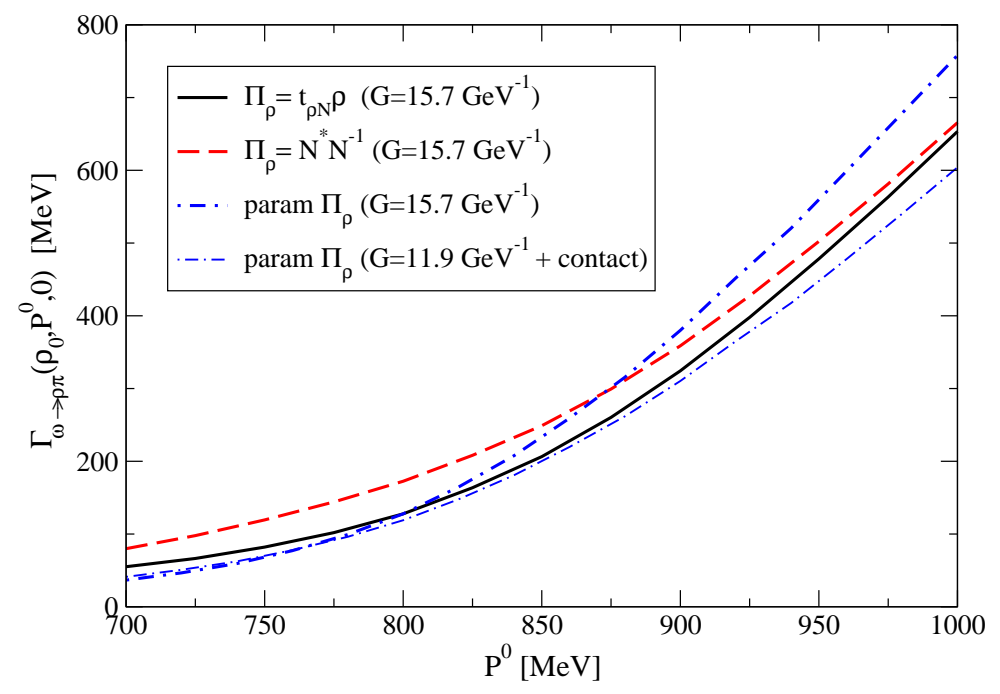

FIG. 10: Width of the $\omega$ meson at normal nuclear matter density and $\vec{P}=0$ as a function of its energy $P^{0}$.

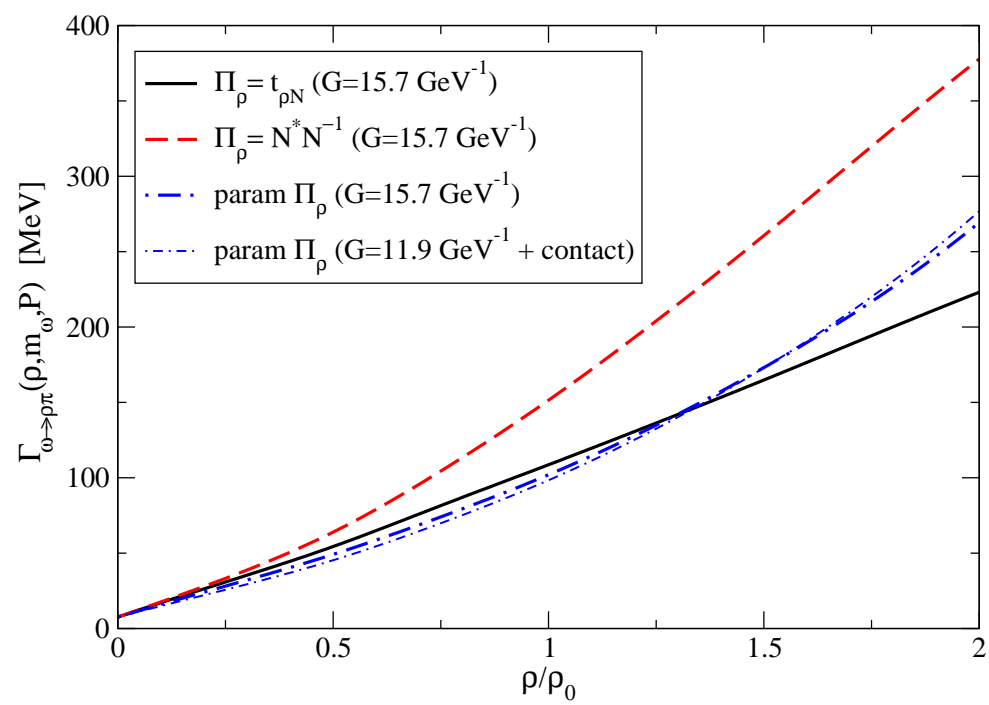

FIG. 11: Width of the omega meson at $P^{0}=m_{\omega}$ and $\vec{P}=0$ as a function of the nuclear matter density. 
In Fig. 10 we show the width of a $\omega$ meson at rest in nuclear matter at normal density as a function of the $\omega$ energy $P^{0}$, for the three prescriptions of the $\rho$-meson self-energy employed in this work and using the model with $G=15.7 \mathrm{GeV}^{-1}$. The in-medium $\omega$ width increases smoothly with energy for all the $\rho$-dressing models employed, the phenomenological one (thick dash-dotted line) presenting a stronger dependence due to the p-wave nature of the employed parametrization, Eq. (17). In this case, results are also shown for the model that uses $G=11.9 \mathrm{GeV}^{-1}$ plus a contact $\omega \rightarrow 3 \pi$ term (thin dash-dotted line). We observe that, up to the free $\omega$ mass, $m_{\omega}=783 \mathrm{MeV}$, both models present a similar behavior but beyond this energy the width of the model employing $G=11.9 \mathrm{GeV}^{-1}$ and a contact-term contribution evolves more slowly, an effect tied to the weaker energy dependence of the contact term.

The dependence of the $\omega$ width on the nuclear matter density is shown in Fig. 11, for the three prescriptions of the $\rho$-meson self-energy and employing the model with $G=15.7 \mathrm{GeV}^{-1}$. We observe a smooth increase of the $\omega$ width with density. The different non-linear density effects observed in the $\omega$ width are tied to the particular way the $\rho$-meson is dressed. In general, the models that have a larger $\rho$-meson strength at low energies also leave a stronger imprint on the $\omega$ self-energy, thus making the non-linear density effects to be more emphasized. An exception occurs for the phenomenological parametrization of Eq. (17). In this case, the p-wave nature of the $\rho$ width makes it to increase more rapidly with density, hence magnifying the non-linear density effects of the $\omega$ width. For this approach to the $\rho$ self-energy, we also present in Fig. 11] a calculation for the model that uses $G=11.9 \mathrm{GeV}^{-1}$ and a contact-term, obtaining essentially the same behavior with density as that for the $G=15.7 \mathrm{GeV}^{-1}$ model.

From the low density behavior of the $\omega$ width, we can derive the imaginary part of the scattering length. This is done via the low-density theorem

$$
\Pi_{\omega}(\rho \rightarrow 0)=t_{\omega N \rightarrow \omega N} \rho
$$

Knowing

$$
a_{\omega N}=-\frac{1}{4 \pi} \frac{M_{N}}{M_{N}+m_{\omega}} t_{\omega N \rightarrow \omega N}
$$

with $t_{\omega N \rightarrow \omega N}$ being the amplitude at threshold, and

$$
\Gamma_{\omega}=-\frac{\operatorname{Im} \Pi_{\omega}}{\omega_{\omega}},
$$

we find

$$
\operatorname{Im} a_{\omega N}=\frac{1}{4 \pi} \frac{M_{N}}{M_{N}+m_{\omega}} \frac{m_{\omega} \Gamma_{\omega}}{\rho} .
$$

Taking our results for the $\omega$ width at $\rho=0.1 \rho_{0}$, in the case the $\rho$ meson is dressed from the unitarized $t_{\rho N \rightarrow \rho N}$ amplitude, we obtain

$$
\operatorname{Im} a_{\omega N} \approx 0.39 \mathrm{fm}
$$

which compares favourably with the values $\operatorname{Im} a_{\omega N} \approx 0.31 \mathrm{fm}$ from [49], $\operatorname{Im} a_{\omega N} \approx 0.30$ fm from [37] and $\operatorname{Im} a_{\omega N} \approx 0.20$ fm from [43]. Not surprisingly our value is somewhat bigger since our approach incorporates more decay channels.

We now present in Fig. 12 the in-medium $\omega$ width correction coming from its coupling to $K \bar{K}$ states and their in-medium excitations. We observe that this contribution is very small compared with the values for the $\omega$ width coming from the anomalous decay $\omega \rightarrow \rho \pi$ in nuclear matter. At normal nuclear matter saturation density and energies around the free $\omega$ mass, the in-medium 


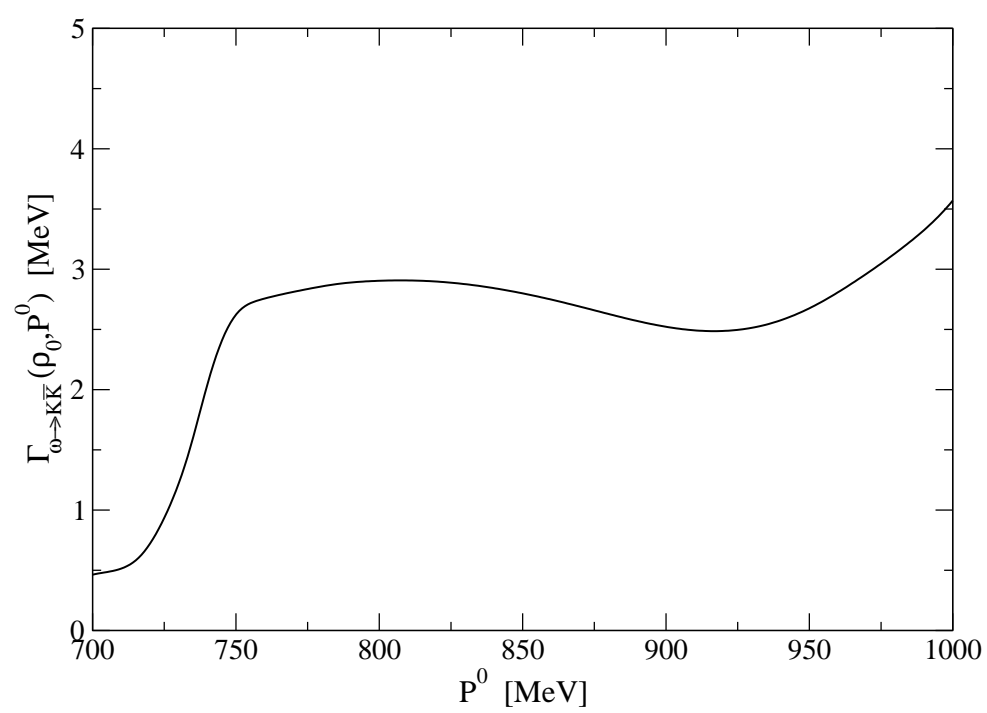

FIG. 12: In-medium $\omega$ width coming from its coupling to $K \bar{K}$, at $\rho_{0}=0.17 \mathrm{fm}^{-3}$ and as a function of the $\omega$ energy $P^{0}$.

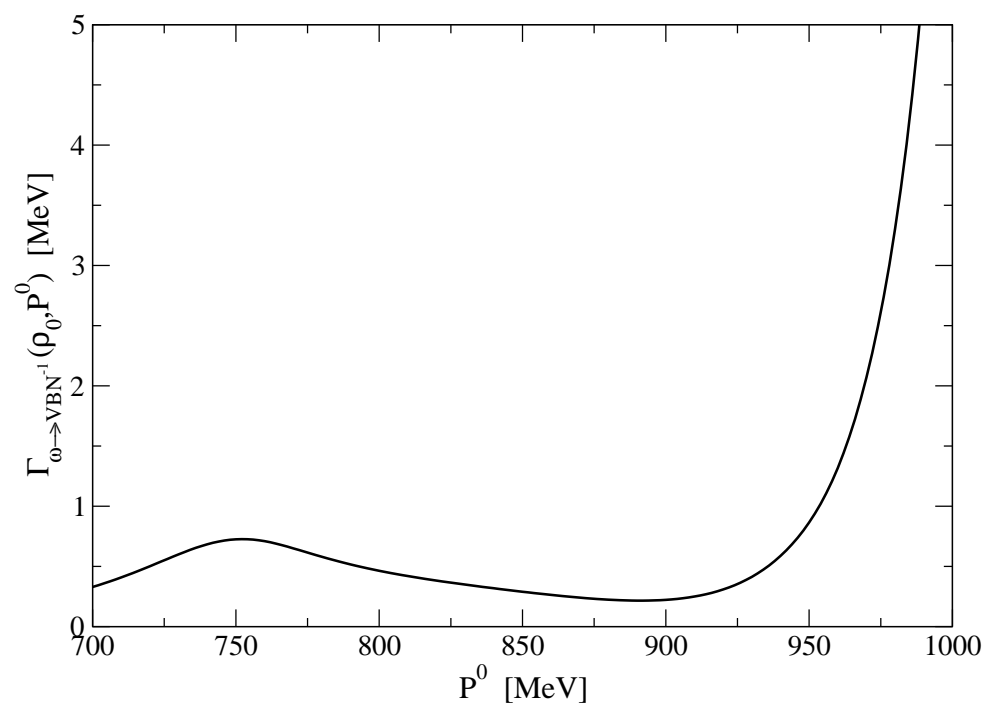

FIG. 13: Width of a zero momentum $\omega$ meson due to the s-wave $\omega N \rightarrow V B$ interaction, at normal nuclear matter density $\rho_{0}=0.17 \mathrm{fm}^{-3}$ and as a function of the $\omega$ energy $P^{0}$.

width correction associated to the $\omega \rightarrow K \bar{K}$ transition for a $\omega$ meson at rest is $\Delta \Gamma_{\omega \rightarrow K \bar{K}}\left(\rho_{0}, m_{\omega}\right)=$ $-\operatorname{Im} \Pi_{\omega \rightarrow K \bar{K}}\left(\rho_{0}, m_{\omega}\right) / m_{\omega}=2.9 \mathrm{MeV}$. This width is mainly coming from the $p$-wave coupling of the antikaon to $Y h$ components, since these are the lowest possible $\bar{K}$ excitations in the medium. This $\omega \rightarrow K \bar{K}$ in-medium width correction is therefore associated to processes such as $\omega N \rightarrow K \Sigma$ and $K \Lambda$, which are above their thresholds by $36 \mathrm{MeV}$ and $110 \mathrm{MeV}$, respectively. The antikaon-nucleon s-wave driven interaction terms, such as $\bar{K} N \rightarrow \pi \Lambda, \pi \Sigma$, which are significantly dominant over the p-wave $\bar{K} N \rightarrow Y$ ones, do not contribute to the related $\omega N \rightarrow K \bar{K} N \rightarrow K \pi \Lambda, K \pi \Sigma$ processes, since their corresponding thresholds are closed by $28 \mathrm{MeV}$ and $102 \mathrm{MeV}$, respectively.

We finally present in Fig. 13 the $\omega$ width correction associated to the elastic an inelastic pro- 
cesses implemented by the $s$-wave interaction of $\omega N$ with vector mesons and baryons as a function of the $\omega$ energy. We observe that this contribution produces a very small $\omega$ width correction, $\Delta \Gamma_{\omega \rightarrow V B N^{-1}}\left(\rho_{0}, m_{\omega}\right) \sim 0.5 \mathrm{MeV}$ for energies around the free $\omega$ mass and at normal nuclear saturation density $\rho_{0}$. This result would not change if a self-consistent calculation of the in-medium $t_{\omega N \rightarrow \omega N}$ amplitude, implementing the full width of the $\omega$ meson in the loop function of the $\omega N$ channel, was attempted. The same applies to a possible incorporation of the in-medium width of the $\rho$ meson. This is due to the fact that the diagonal $\omega N-\omega N$ and non-diagonal $\omega N-\rho N$ couplings are zero [55] and any change in the $\omega$ or $\rho$ widths will only affect the $\omega N-\omega N$ amplitude via the indirect coupling to $K^{*} Y(Y=\Lambda, \Sigma)$ states, lying about $300 \mathrm{MeV}$ above the $\omega N$ threshold, in higher order scattering processes. The small $\omega$ width correction obtained from the $s$-wave interaction of the vector mesons and baryons should be associated to the elastic $\omega N \rightarrow \omega N$ and the inelastic $\omega N \rightarrow \rho N$ processes, which are the only possible decay channels allowed in the employed vector-baryon interaction model [55].

The implementation of pseudoscalar mesons into the scheme, hence opening vector-baryon to pseudoscalar-baryon transitions such as $\omega N \rightarrow \pi N$, does not seem to change the $t_{\omega N \rightarrow \omega N}$ amplitude significantly [71]. We note that the coupling between the vector-baryon and the pseudoscalarbaryon channels implemented by the box-diagrams built up in [1] is done through t-channel pseudoscalar meson exchange, while the anomalous t-channel vector-exchange terms are not considered. Actually, a proper implementation in the $\omega \rightarrow \rho \pi$ decay channel of the low energy branch of the $\rho$-meson self-energy, associated to $p h$ excitations via a $\rho N N$-type coupling, would provide these type of terms, giving rise to an additional contribution to the $\omega$ width coming from the $\omega N \rightarrow \pi N$ transition. However, our simple parametrization of the $\rho$-meson self-energy, given in Eq. (17), or the other two prescriptions miss this source of low energy strength of the $\rho$ spectral function. For this reason, we adopt the pragmatic, safe and model independent point of view discussed by Friman in [91], where the contribution of the $\pi N$ channel to the width of the $\omega$ meson was obtained from the use of detailed balance (to relate the measured $\pi^{-} p \rightarrow \omega n$ cross section to the one of the reversed process $\omega n \rightarrow \pi^{-} p$ ) and unitarity (to connect the value of this cross section with the $\pi^{-} p$ contribution to the $\omega n$ forward scattering amplitude). A fit to the $\pi^{-} p \rightarrow \omega n$ data provided a $\pi N$ channel contribution to the width of the $\omega$ meson of about $9 \mathrm{MeV}$ [91].

Joining all the contributions together, we conclude that the width of the $\omega$ meson at rest in nuclear matter at normal saturation density is $\Gamma_{\omega}\left(\rho_{0}, m_{\omega}\right)=7.6 \mathrm{MeV}$ (free width) $+108.7 \mathrm{MeV}$ $(\omega N \rightarrow \pi \pi N, \omega N N \rightarrow \pi N N)+2.9 \mathrm{MeV}(\omega N \rightarrow K Y)+0.5 \mathrm{MeV}\left(\omega N \rightarrow K^{*} Y \rightarrow \rho N\right)+9 \mathrm{MeV}$ $(\omega N \rightarrow \pi N)=129 \pm 10 \mathrm{MeV}$, where the $10 \mathrm{MeV}$ error is associated to uncertainties of the theoretical model. We note that one could add one more $\mathrm{MeV}$ to account for the other free decay channels of the $\omega$ meson, $\omega \rightarrow \pi^{0} \gamma$ and $\omega \rightarrow \pi^{+} \pi^{-}$.

Our calculated value of the width of the $\omega$ meson at rest in nuclear matter is larger than that found by other works in the literature. A few models derive the $\omega$ width from the elastic $\omega N$ amplitude by means of the low-density theorem, finding $\omega$ width values of the order of $40-60 \mathrm{MeV}$ [37, 43, 49] up to $75 \mathrm{MeV}$ [52]. The works of Refs. [43, 49] employ amplitudes that have been fitted to a variety of elastic and inelastic $\gamma N$ and $\pi N$ data, implementing resonance contributions and rescattering effects in unitary models. The self-consistent approach of Ref. [46], studying the influence of the in-matter spectral function of the pion in the vector-meson self-energies, is the one closer to the methodology employed in the present work for the contribution to the $\omega$ width from the $\rho \pi$ mechanism. Yet, a value of about $60 \mathrm{MeV}$ is quoted in Ref. [46] while our result from this $\omega \rightarrow \rho \pi$ channel is of the order of $100 \mathrm{MeV}$. We have traced back the differences to the much softer form-factor of gaussian type, $F^{\mathrm{g}}(\vec{q})=\exp \left(-\vec{q}^{2} / \Lambda^{2}\right)$ with $\Lambda=440 \mathrm{MeV}$, employed in Ref. [46], compared with the monopole form-factor, $F^{\mathrm{m}}\left(\vec{q}^{2}\right)=\left(\Lambda_{\pi}^{2}-m_{\pi}^{2}\right) /\left[\Lambda_{\pi}^{2}-q^{02}+\vec{q}^{2}\right]$ with $\Lambda_{\pi}=1200 \mathrm{MeV}$, employed in the present work and taken from $N N$ interaction studies [67]. The ratio $\left(F^{\mathrm{m}} / F^{\mathrm{g}}\right)^{2}$ depends on the loop momentum over which one must integrate to obtain the $\omega$ 
width but, for a characteristic value of $\vec{q}=250 \mathrm{MeV} / \mathrm{c}$ and setting $q^{0}=0 \mathrm{MeV}$, it amounts to 1.7 , explaining the larger size of the $\omega$ width obtained in the present work. Large values of the width are also reported in [70], associated to the large modification of the $\rho$ spectral function at low invariant masses. We showed before that, when a microscopical model for the $t_{\rho N \rightarrow \rho N}$ amplitudes is used to evaluate the $\rho$ self-energy, this contribution is more moderate.

In order to compare with the experimental determination of the $\omega$ width, we need to extend our calculation to finite momentum. Although, in principle, the $P^{0}$ and $\vec{P}$ variables of the $\omega$ meson are independent in nuclear matter, one must correlate them on-shell if one wishes to compare with the results of the $\omega$ in-medium width of [31], since the $\omega$ detection is done imposing the invariant mass of the $\pi^{0} \gamma$ system to match the $\omega$ mass. In Fig. 14 we show the momentum dependence of the $\omega$ width coming from the $\omega \rightarrow 3 \pi$ channel in nuclear matter at saturation density, for the three different prescriptions of the $\rho$-meson self-energy employed in this work. We observe that $\Gamma_{\omega \rightarrow 3 \pi}$ rises smoothly with momentum, and it can reach values of about $200 \mathrm{MeV}$ at $P=600 \mathrm{MeV} / \mathrm{c}$. We should keep in mind that we must add about $15 \mathrm{MeV}$ to these numbers from the other sources discussed above. The experimental width is quoted to be $\Gamma_{\omega} \approx 130-150 \mathrm{MeV}$ for an average 3 -momentum of $1.1 \mathrm{GeV} / \mathrm{c}$ [31]. Apart from this global average, which is in qualitative agreement with what we obtain, the experimental analysis also reports values of $\Gamma_{\omega}$ for different momentum bins. One then sees that we obtain a good agreement within errors for the lower two momentum values reported in Fig. 4 of Ref. [31], $400 \mathrm{MeV} / \mathrm{c}$ and $600 \mathrm{MeV} / \mathrm{c}$, where our results should be more accurate.

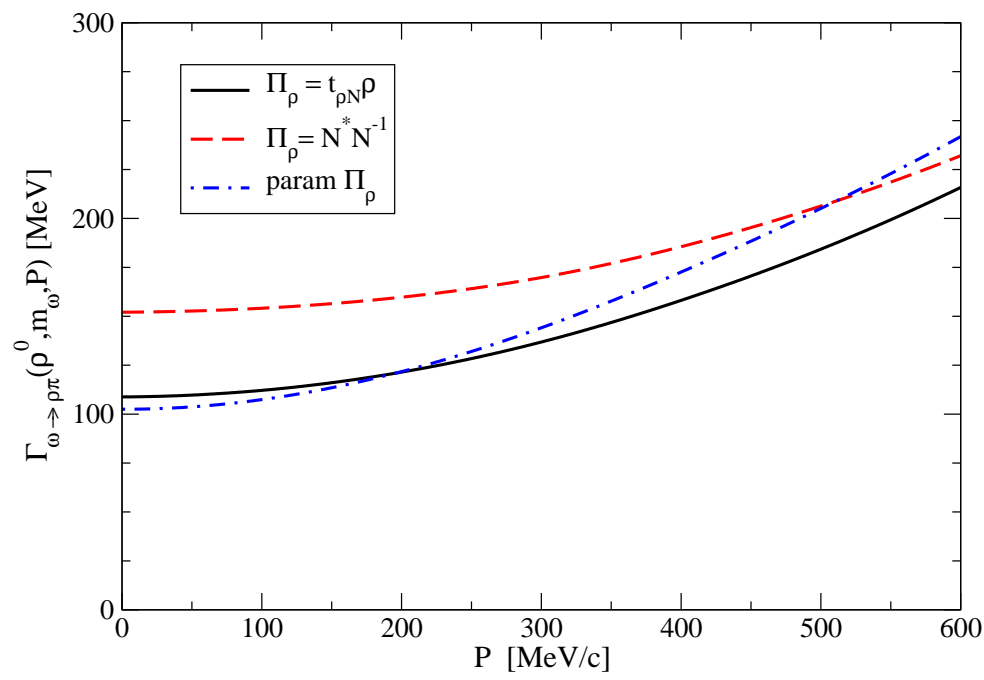

FIG. 14: Contribution to the $\omega$ width coming from the $\omega \rightarrow 3 \pi$ channel in nuclear matter at saturation density, as a function of momentum, for the three different prescriptions of the $\rho$-meson self-energy employed in this work.

Before closing this section, we note that the theoretical study of [49] shows a somewhat stronger momentum dependence of the transverse component of the $\omega$ self-energy and, consequently, the width evolves from a value of $60 \mathrm{MeV}$ at zero momentum to around $130 \mathrm{MeV}$ at $600 \mathrm{MeV} / \mathrm{c}$, a value that is roughly consistent with the experimental results [31]. The new light brought into this problem by the present work allows us to examine the results of [49] with a new perspective. We recall that in that work the $\omega$ width is obtained in a $t_{\omega N \rightarrow \omega N} \rho$ approximation from the elastic $\omega N$ amplitude, which is found to be dominated by resonance hole components, the $1 / 2^{-} S_{11}(1535)$ playing the most important role. In the present work, the main contribution to the $\omega$ width comes from the $\omega \rightarrow \rho \pi$ channel, when the $\pi$ and $\rho$ mesons are allowed to be dressed in the medium, 
which incorporates terms of higher orders in density. This $\omega \rightarrow \rho \pi$ process would correspond to a background type term in [49], claimed to be less important than the resonance hole contributions. We believe that the combination of a varied number of resonances with a priori unknown couplings that are fitted to inelastic $\pi N \rightarrow \omega N$ and $\gamma N \rightarrow \omega N$ processes brings uncertainties to the elastic $\omega N \rightarrow \omega N$ amplitudes, particularly when it comes to the coupling to $\omega N$ states of a resonance like the $S_{11}(1535)$ which is quite below the $\omega N$ threshold. In our approach, the resonant contributions to the $\omega N$ amplitude are generated from a unitary model in coupled channels, including also $\rho N$, $\phi N, K^{*} \Lambda$ and $K^{*} \Sigma$, and using the dynamics of the hidden gauge Lagrangians. The $S_{11}(1535)$ is not obtained from the vector-baryon dynamics but it comes out from the interaction of the pseudoscalar-baryon $\pi N, \eta N, K \Lambda$ and $K \Sigma$ channels [76, 92]. One can consider vector-baryon and pseudoscalar-baryon components together, as done in [71], but the mixing is small and the different dynamically generated states basically keep their original identity. Yet, it is simple to determine the coupling of one particular pseudoscalar-baryon resonance to a vector-baryon channel using chiral unitary methods and vector meson dynamics, as done in [93]. Neglecting terms of order $\mathcal{O}\left((\vec{q} / 2 M)^{2}\right)$, the coupling of the $N^{*}(1535)$ to $\omega N$ states was found to be given by

$$
-i t_{N^{*} N \omega}=g_{N^{*} N \omega} \vec{\sigma} \vec{\epsilon}
$$

where $\vec{\epsilon}$ is the $\omega$ polarization (the zeroth component $\epsilon^{0}$ was neglected since small momenta of the $\omega$ meson were being considered). The dynamical model of Ref. [93] determined the value $g_{N^{*} N \omega}=0.02+i 0.28$. It is straightforward to show that, neglecting terms of $\mathcal{O}\left((\vec{q} / 2 M)^{2}\right)$, the Lagrangian employed in Ref. [49],

$$
\mathcal{L}_{1 / 2^{-} N \omega}=i \bar{u}_{R} \gamma_{5}\left(g_{1} \gamma^{\mu} \omega_{\mu}-\frac{g_{2}}{2 M_{N}} \sigma^{\mu \nu} \partial_{\nu} \omega_{\mu}\right) u_{N}
$$

produces a term equivalent to that of Eq. (47), as long as one identifies

$$
g_{N^{*} N \omega}=-g_{1}+g_{2} \frac{m_{\omega}}{2 M_{N}} .
$$

Taking the values $g_{1}=3.79$ and $g_{2}=6.50$ quoted in [49], one obtains $g_{N^{*} N \omega}=1.09$. Despite the considerable cancellation between the $g_{1}$ and $g_{2}$ terms, this value is still much larger than the absolute value of the coupling derived in [93]. Consequently, the contribution of the $S_{11}(1535)$ to the $\omega$ width turns out to be about 15 times larger in [49] than that one would obtain using the coupling derived in [93]. This is just a simple example trying to show that the couplings of some resonances to $\omega N$ states at threshold might not be very well constrained experimentally due to interferences between various a priori unknown resonant terms and background contributions.

\section{CONCLUSIONS}

We have evaluated the width of the $\omega$ meson in the nuclear medium, from a variety of processes.

We have first considered the free decay mode of the $\omega$ into three pions, which is dominated by $\rho \pi$ decay, and, by replacing the $\rho$ and $\pi$ propagators by their medium modified ones, we have obtained the medium corrections to the $\omega$ width. In order to get a better feeling of uncertainties and place the results in consistency with advances made recently on the theoretical description of the $\rho N$ interaction, we have employed three different models for the $\rho$-meson propagator. We have considered a phenomenological approach consisting in parameterizing the $\rho$ spectral function by simply increasing the $\rho$ width by $33 \%$ at normal nuclear matter density. We have also taken a more realistic approach that implements the coupling of the $\rho$ to an explicit $N^{*}(1520) h$ excitation, hence increasing the $\rho$ strength at low invariant masses. Finally, we have implemented a recently derived 
$\rho N$ scattering amplitude, based on coupled-channel unitarizing techniques using local hidden gauge Lagrangians. The results of the three approaches are qualitatively similar, but our analysis is useful to show the relevance of the role of the resonances like the $N^{*}(1520)$, which are dynamically generated in the latter approach. We establish the relevance of this resonance on the in-medium width of the $\omega$ meson and shed light on results obtained with approaches that incorporate it more phenomenologically. We have also taken two different prescriptions for the $\omega \rho \pi$ coupling $G$, a value adjusted to reproduce vector meson radiative decays, in which case the model must be supplemented by the additional consideration of a contact term, or an effective coupling adjusted to reproduce the free decay width of the $\omega$ meson into three pions. Both prescriptions give rise to very similar results for the in-medium $\omega$ width.

We have also taken into account the contributions from the virtual $\omega \rightarrow K \bar{K}$ decay channel, which is open in the medium due to the excitation of $Y h$ components with antikaon quantum numbers. This $\omega$ decay channel has provided a very small contribution to the $\omega$ decay width, less than $3 \mathrm{MeV}$, because the dominant s-wave components of the $\bar{K} N$ interaction, leading to $\omega N \rightarrow$ $K Y \pi$ transitions, are not energetically allowed. The only possible transitions are $\omega N \rightarrow K Y$, which are triggered by the much weaker p-wave component of the $\bar{K} N$ interaction at the low energies explored by the processes studied here.

Finally, we have obtained the quasielastic and inelastic mechanisms induced by a $t_{\omega N \rightarrow \omega N} \rho$ term, where $t_{\omega N \rightarrow \omega N}$ is the $\omega N$ scattering matrix evaluated within a unitary scheme in coupled channels of vector-baryon type. This gives rise to a practically negligible contribution, of $0.5 \mathrm{MeV}$, due to the zero value of the tree-level $\omega N-\omega N$ and $\omega N-\rho N$ amplitudes of the employed model. Unitarization through the coupling to $K^{*} Y$ channels gives a non-zero but small value for these amplitudes. The extension of the model to incorporate the pseudoscalar-baryon channels through box diagrams employing normal VPP couplings does not change the situation significantly. We have therefore added a $9 \mathrm{MeV}$ contribution to the $\omega$ width from the $\omega N \rightarrow \pi N$ processes, following a model independent approach based on detailed balance and unitarity.

With all these contributions, we obtain a value of $129 \pm 10 \mathrm{MeV}$ for the width of the $\omega$ meson at rest in nuclear matter at normal saturation density, which is a substantially larger width than that quoted in previous theoretical works, many of them based on an indirect extraction of the $\omega N$ scattering amplitude from fits to $\gamma N \rightarrow \omega N, \pi N \rightarrow \omega N, \omega N \rightarrow \pi \pi N$ data.

We have also evaluated the momentum dependence of the in-medium $\omega$ width and show that it increases with momentum, being about $200 \mathrm{MeV}$ at $600 \mathrm{MeV} / \mathrm{c}$. The range of values that we obtain up to $600 \mathrm{MeV} / \mathrm{c}$ are in line with the width of the order of 130-150 MeV obtained by the CBELSA/TAPS collaboration in photoproduction reactions off nuclei for an average momentum of around $1.1 \mathrm{GeV} / \mathrm{c}$. More specifically, we obtain a very good agreement, within errors, with the $\Gamma_{\omega}$ results reported for the two lower momentum bins of $400 \mathrm{MeV} / \mathrm{c}$ and $600 \mathrm{MeV} / \mathrm{c}$.

\section{Acknowledgments}

We would like to acknowledge useful discussions with U. Mosel. This work is partly supported by the Spanish Ministerio de Economia y Competitividad and European FEDER funds under the contract numbers FIS2011-28853-C02-01, FIS2011-24154, the Generalitat Valenciana in the program Prometeo, 2009/090 and Grant No. 2009SGR-1289 from Generalitat de Catalunya. L.T. acknowledges support from Ramon y Cajal Research Programme, and from FP7-PEOPLE2011-CIG under contract PCIG09-GA-2011-291679. We acknowledge the support of the European Community-Research Infrastructure Integrating Activity Study of Strongly Interacting Matter (acronym HadronPhysics3, Grant Agreement n. 283286) under the Seventh Framework Programme 
of EU.

[1] R. Rapp and J. Wambach, Adv. Nucl. Phys. 25, 1 (2000)

[2] R. S. Hayano and T. Hatsuda, Rev. Mod. Phys. 82, 2949 (2010).

[3] S. Leupold, V. Metag and U. Mosel, Int. J. Mod. Phys. E 19, 147 (2010).

[4] E. Oset, A. Ramos, E. J. Garzon, R. Molina, L. Tolos, C. W. Xiao, J. J. Wu and B. S. Zou, Int. J. Mod. Phys. E 21, 1230011 (2012).

[5] M. Bando, T. Kugo, S. Uehara, K. Yamawaki and T. Yanagida, Phys. Rev. Lett. 54, 1215 (1985).

[6] M. Bando, T. Kugo and K. Yamawaki, Phys. Rept. 164, 217 (1988).

[7] U. G. Meissner, Phys. Rept. 161, 213 (1988).

[8] M. Harada and K. Yamawaki, Phys. Rept. 381, 1 (2003).

[9] R. Rapp, G. Chanfray and J. Wambach, Nucl. Phys. A 617, 472 (1997).

[10] W. Peters, M. Post, H. Lenske, S. Leupold and U. Mosel, Nucl. Phys. A 632, 109 (1998).

[11] R. Rapp and J. Wambach, Adv. Nucl. Phys. 25, 1 (2000).

[12] M. Urban, M. Buballa, R. Rapp and J. Wambach, Nucl. Phys. A 673, 357 (2000).

[13] D. Cabrera, E. Oset and M. J. Vicente Vacas, Nucl. Phys. A 705, 90 (2002).

[14] D. Cabrera and M. J. Vicente Vacas, Phys. Rev. C 67, 045203 (2003).

[15] D. Cabrera, L. Roca, E. Oset, H. Toki and M. J. Vicente Vacas, Nucl. Phys. A 733, 130 (2004).

[16] S. Damjanovic et al. [NA60 Collaboration], Nucl. Phys. A 783, 327 (2007) .

[17] M. H. Wood et al. [CLAS Collaboration], Phys. Rev. C 78, 015201 (2008).

[18] K. Ozawa et al. [E325 Collaboration], Phys. Rev. Lett. 86, 5019 (2001).

[19] T. Tabaru, H. En'yo, R. Muto, M. Naruki, S. Yokkaichi, J. Chiba, M. Ieiri and O. Sasaki et al., Phys. Rev. C 74, 025201 (2006).

[20] F. Sakuma et al. [E325 Collaboration], Phys. Rev. Lett. 98, 152302 (2007).

[21] M. H. Wood et al. [CLAS Collaboration], Phys. Rev. Lett. 105, 112301 (2010).

[22] D. Trnka et al. [CBELSA/TAPS Collaboration], Phys. Rev. Lett. 94, 192303 (2005).

[23] M. Kaskulov, E. Hernandez and E. Oset, Eur. Phys. J. A 31, 245 (2007).

[24] M. Nanova et al. [CBELSA/TAPS Collaboration], Phys. Rev. C 82, 035209 (2010).

[25] V. Metag, Talk at the Workshop on Electromagnetic Probes of Strongly Interacting Matter: Status and Future of Low-Mass Lepton-Pair Spectroscopy, ECT*, Trento (Italy), 20-24 May, 2013.

[26] D. Trnka, PhD Thesis, University of Giessen (Germany).

[27] V. Metag, Prog. Theor. Phys. Suppl. 168, 503 (2007).

[28] M. Kaskulov, H. Nagahiro, S. Hirenzaki and E. Oset, Phys. Rev. C 75, 064616 (2007).

[29] V. Metag, Prog. Part. Nucl. Phys. 61, 245 (2008).

[30] M. Kaskulov, E. Hernandez and E. Oset, Eur. Phys. J. A 46, 223 (2010).

[31] M. Kotulla et al. [CBELSA/TAPS Collaboration], Phys. Rev. Lett. 100, 192302 (2008).

[32] H. C. Jean, J. Piekarewicz and A. G. Williams, Phys. Rev. C 49, 1981 (1994).

[33] F. Klingl, N. Kaiser and W. Weise, Nucl. Phys. A 624, 527 (1997).

[34] K. Saito, K. Tsushima, A. W. Thomas and A. G. Williams, Phys. Lett. B 433, 243 (1998).

[35] K. Tsushima, D. -H. Lu, A. W. Thomas and K. Saito, Phys. Lett. B 443, 26 (1998).

[36] B. Friman, Acta Phys. Polon. B 29, 3195 (1998).

[37] F. Klingl, T. Waas and W. Weise, Nucl. Phys. A 650, 299 (1999).

[38] M. Post and U. Mosel, Nucl. Phys. A 688, 808 (2001).

[39] K. Saito, K. Tsushima, D. -H. Lu and A. W. Thomas, Phys. Rev. C 59, 1203 (1999).

[40] G. I. Lykasov, W. Cassing, A. Sibirtsev and M. V. Rzyanin, Eur. Phys. J. A 6, 71 (1999).

[41] A. Sibirtsev, C. Elster and J. Speth, nucl-th/0203044.

[42] A. K. Dutt-Mazumder, R. Hofmann and M. Pospelov, Phys. Rev. C 63, 015204 (2001).

[43] M. F. M. Lutz, G. Wolf and B. Friman, Nucl. Phys. A 706, 431 (2002) [Erratum-ibid. A 765, 431 (2006)].

[44] S. Zschocke, O. P. Pavlenko and B. Kampfer, Phys. Lett. B 562, 57 (2003).

[45] A. K. Dutt-Mazumder, Nucl. Phys. A 713, 119 (2003).

[46] F. Riek and J. Knoll, Nucl. Phys. A 740, 287 (2004). 
[47] P. Muhlich, T. Falter and U. Mosel, Eur. Phys. J. A 20, 499 (2004).

[48] F. Eichstaedt, S. Leupold, U. Mosel and P. Muehlich, Prog. Theor. Phys. Suppl. 168, 495 (2007).

[49] P. Muehlich, V. Shklyar, S. Leupold, U. Mosel and M. Post, Nucl. Phys. A 780, 187 (2006).

[50] P. Muhlich and U. Mosel, Nucl. Phys. A 773, 156 (2006).

[51] B. Steinmueller and S. Leupold, Nucl. Phys. A 778, 195 (2006).

[52] A. T. Martell and P. J. Ellis, Phys. Rev. C 69, 065206 (2004).

[53] S. Ghosh and S. Sarkar, Eur. Phys. J. A 49, 97 (2013).

[54] S. Sarkar, B. -X. Sun, E. Oset et al., Eur. Phys. J. A 44, 431-443 (2010).

[55] E. Oset and A. Ramos, Eur. Phys. J. A 44, 445 (2010).

[56] L. Tolos, R. Molina, E. Oset and A. Ramos, Phys. Rev. C 82, 045210 (2010).

[57] J. Beringer et al. [Particle Data Group Collaboration], Phys. Rev. D 86, 010001 (2012).

[58] A. Bramon, A. Grau and G. Pancheri, Phys. Lett. B 283, 416 (1992).

[59] G. Ecker, J. Gasser, A. Pich and E. de Rafael, Nucl. Phys. B 321, 311 (1989).

[60] G. Ecker, J. Gasser, H. Leutwyler, A. Pich and E. de Rafael, Phys. Lett. B 223, 425 (1989).

[61] M. Benayoun, P. David, L. DelBuono and O. Leitner, Eur. Phys. J. C 65, 211 (2010).

[62] H. Nagahiro, L. Roca, A. Hosaka and E. Oset, Phys. Rev. D 79, 014015 (2009).

[63] J. E. Palomar and E. Oset, Nucl. Phys. A 716, 169 (2003).

[64] G. Ecker, Prog. Part. Nucl. Phys. 35, 1 (1995).

[65] V. Bernard, N. Kaiser and U. G. Meissner, Int. J. Mod. Phys. E 4, 193 (1995).

[66] E. Oset, H. Toki and W. Weise, Phys. Rept. 83, 281 (1982).

[67] R. Machleidt, K. Holinde and C. Elster, Phys. Rept. 149, 1 (1987).

[68] E. Oset, P. Fernandez de Cordoba, L. L. Salcedo and R. Brockmann, Phys. Rept. 188, 79 (1990).

[69] A. Ramos, E. Oset and L. L. Salcedo, Phys. Rev. C 50, 2314 (1994).

[70] D. Cabrera and R. Rapp, arXiv:1307.4001 [nucl-th].

[71] E. J. Garzon and E. Oset, Eur. Phys. J. A 48, 5 (2012).

[72] E. J. Garzon, J. J. Xie and E. Oset, Phys. Rev. C 87, 055204 (2013).

[73] D. García-Gudino and G. Toledo-Sanchez, Int. J. Mod. Phys. A 27, 1250101 (2012).

[74] E. Oset and A. Ramos, Nucl. Phys. A 635, 99 (1998).

[75] A. Ramos, E. Oset and C. Bennhold, Phys. Rev. Lett. 89, 252001 (2002).

[76] T. Inoue, E. Oset and M. J. Vicente Vacas, Phys. Rev. C 65, 035204 (2002).

[77] J. A. Oller and U. G. Meissner, Phys. Lett. B 500, 263 (2001).

[78] A. Ramos and E. Oset, Nucl. Phys. A 671, 481 (2000).

[79] L. Tolos, A. Ramos and E. Oset, Phys. Rev. C 74, 015203 (2006).

[80] D. Cabrera, A. Polls, A. Ramos and L. Tolos, Phys. Rev. C 80, 045201 (2009).

[81] T. Waas, N. Kaiser and W. Weise, Phys. Lett. B 379, 34 (1996).

[82] T. Waas and W. Weise, Nucl. Phys. A 625, 287 (1997).

[83] C. Garcia-Recio, J. Nieves, T. Inoue and E. Oset, Phys. Lett. B 550, 47 (2002).

[84] E. Oset and A. Ramos, Nucl. Phys. A 679, 616 (2001).

[85] M. Herrmann, B. L. Friman and W. Norenberg, Nucl. Phys. A 560, 411 (1993).

[86] G. Chanfray and P. Schuck, Nucl. Phys. A 555, 329 (1993).

[87] L. Roca, E. Oset and J. Singh, Phys. Rev. D 72, 014002 (2005).

[88] H. Nagahiro, L. Roca and E. Oset, Eur. Phys. J. A 36, 73 (2008).

[89] A. Martinez Torres, K. P. Khemchandani and E. Oset, Phys. Rev. C 77, 042203 (2008).

[90] K. P. Khemchandani, A. Martinez Torres and E. Oset, Eur. Phys. J. A 37, 233 (2008).

[91] B. Friman, in Proceedings of the APCTP Workshop on astro-hadron physics: Properties of hadrons in matter, edited by G.E. Brown, C.H. Lee, H.K. Lee, D.P. Min (World Scientific, Singapore, 1999), p 337.

[92] N. Kaiser, P. B. Siegel and W. Weise, Phys. Lett. B 362, 23 (1995).

[93] M. Doring, E. Oset and B. S. Zou, Phys. Rev. C 78, 025207 (2008).

[94] J. A. Gomez Tejedor and E. Oset, Nucl. Phys. A 571, 667 (1994).

[95] J. A. Gomez Tejedor and E. Oset, Nucl. Phys. A 600, 413 (1996). 